EPJ Web of Conferences 18, 02003 (2011)

DOI: $10.1051 /$ epjconf/20111802003

(C) Owned by the authors, published by EDP Sciences, 2011

\title{
Gas phase reactive collisions, experimental approach
}

\author{
A. Canosa ${ }^{\mathrm{a}}$ \\ Institut de Physique de Rennes, UMR 6251 CNRS-Université, Équipe Astrochimie \\ Expérimentale, Bât. 11 C, Campus de Beaulieu, Université de Rennes 1, \\ 35042 Rennes Cedex, France
}

\begin{abstract}
Since 1937 when the first molecule in space has been identified, more than 150 molecules have been detected. Understanding the fate of these molecules requires having a perfect view of their photochemistry and reactivity with other partners. It is then crucial to identify the main processes that will produce and destroy them. In this chapter, a general view of experimental techniques able to deliver gas phase chemical kinetics data at low and very low temperatures will be presented. These techniques apply to the study of reactions between neutral reactants on the one hand and reactions involving charge species on the other hand.
\end{abstract}

\section{INTRODUCTION}

One of the main stakes of modern astrophysics certainly lies in having a perfect understanding of the birth of stars and their related planetary systems. Dense interstellar clouds are presently considered as the cradles of stars and the molecules that are observed in these environments play a significant role in their dynamical stability. For example, they represent important cooling sources by converting kinetic energy into radio or $\mathrm{mm}$ radiations after having suffered inelastic collisions with other species [1]. The emitted radiations from the resulting excited molecules can escape from the cloud and allows us to detect their spectroscopic signature using ground-based telescopes or space observatories. Molecular cooling lowers the thermal pressure in the clouds which eventually will collapse under gravitation forces [2]. Understanding the fate of interstellar molecules therefore, is of major interest and this requires considering carefully their photochemistry in every astrophysical environment.

The methylidyne radical $\mathrm{CH}$ was the first detected molecule in space in 1937. Since then, more than 150 molecules have been identified (>200 including isotopologues) among them about 50 in comets and 30 in the different bodies of the solar system [3]. The molecules range in size from diatomics to cyanodecapentayne $\mathrm{HC}_{11} \mathrm{~N}$, a thirteen-atom nitrile from the family of cyanopolyynes. Among these, carbon-containing molecules are greatly dominant and most of the organic species that have been observed are very unsaturated such as the radical $\mathrm{C}_{\mathrm{n}} \mathrm{H}(\mathrm{n}=1$ to 8$)$ or $\mathrm{HC}_{\mathrm{n}} \mathrm{N}(\mathrm{n}=1$ to 5 and 7,9 , 11) series. Several cyclic species have been identified such as for instance $c-\mathrm{C}_{3} \mathrm{H}, \mathrm{c}-\mathrm{SiC}_{3}, \mathrm{c}-\mathrm{C}_{3} \mathrm{H}_{2}$, c- $\mathrm{CH}_{2} \mathrm{OCH}_{2}$ or benzene $\mathrm{C}_{6} \mathrm{H}_{6}$ but until recently the search for polycyclic aromatic hydrocarbons (PAHs) remained unsuccessful. In 2008, however, detection of the naphthalene cation $\mathrm{C}_{10} \mathrm{H}_{8}^{+}$was claimed [4] in either a relatively diffuse interstellar or circumstellar environment in the direction of the Perseus complex. In 2010, the same group, in the same direction, found evidence for the presence of the anthracene cation $\mathrm{C}_{14} \mathrm{H}_{10}^{+}$[5]. Other cations have also been detected in space. Although they only represent about $10 \%$ of the known molecules, they play a major role in the chemistry in space. Recently, the discovery of several anions $\mathrm{C}_{n} \mathrm{H}^{-}\left(\mathrm{n}=4,6\right.$ and 8) and $\mathrm{C}_{\mathrm{n}} \mathrm{N}^{-}(\mathrm{n}=1 ; 3$ and probably 5) in the

\footnotetext{
ae-mail: andre.canosa@univ-rennes1.fr
}

This is an Open Access article distributed under the terms of the Creative Commons Attribution-Noncommercial License 3.0, which permits unrestricted use, distribution, and reproduction in any noncommercial medium, provided the original work is properly cited. 
interstellar medium and evidence for the presence of very large negatively charged molecules in the atmosphere of Titan shed new lights on the chemistry of astrophysical environments.

The variety, complexity and sometimes exoticism of the molecules presently discovered in extraterrestrial environments arouse a lot of interrogations in the scientific community. Among them the identification of the mechanisms of formation and destruction of these species is of vital importance for the understanding of the chemical evolution of astrophysical bodies. This is why photochemical models of the interstellar medium [6,7] and also planetary atmospheres [8-14] have been developed in the last four decades to try to find chemical routes and schemes that are able to explain the astrophysical observations. These models require a large amount of data specific to the environment of interest. This includes the flux of photons and cosmic rays and also the rate coefficients and branching ratios for all chemical reactions that can play a role in the medium. Presently, the more sophisticated models gather about 400 different species and 5000 chemical and photophysical processes $[6,15]$. The principal networks are issued from the Ohio State University (USA) [16] and from the UMIST (University of Manchester Institute of Science and Technology, GB) [17] and are available on the web where they are updated regularly. These networks have been developed for modelling the various interstellar environments including dense and diffuse clouds, circumstellar envelopes, hot cores, protostellar environments etc. Photochemical models that have been constructed to match the observations in planetary atmospheres, however, are smaller in size because the number of molecules, that have been identified there, is significantly less than in interstellar space. It is worthwhile mentioning here that about only one third of the included processes has been the subject of laboratory studies either experimental or theoretical and just a small fraction of these do provide information on the temperature dependence over a significant range compatible with the physical conditions reigning in space and planetary atmospheres. Interestingly, the low densities that prevail in the interstellar medium restrict the chemistry to two-body reactions whereas three-body processes (i.e.: pressure dependent) can also play a role in planetary atmospheres. Secondly, cold temperatures are characteristic of most of these environments excepting shock interstellar regions and the inner part of circumstellar envelopes. As a consequence, only exothermic processes can take place in these media (especially dense clouds) and any activation barrier along the potential energy surface of a given reaction will eliminate this one from the chemical network. Laboratory astrophysics and more particularly temperature dependent studies are therefore of fundamental interest to provide this kind of information together with the determination of rate coefficients and branching ratios.

As mentioned in other chapters, hydrogen is the dominant species in space. It can be found in atomic or molecular form according to the penetration depth of UV radiations which is essentially dependent on the density of the medium. On another side, cosmic ray bombardment has to be taken into account in all environments. Therefore, basically and quite schematically, the chain of reactions in interstellar clouds starts with the ionisation of $\mathrm{H}, \mathrm{He}$ and $\mathrm{C}$ atoms mainly and molecular hydrogen in denser regions. Then, a series of ion-atom and ion-molecule reactions for which $\mathrm{H}_{3}^{+}$is a major partner, lead to a variety of complex molecular ions. Eventually, dissociative recombination processes produce neutral fragments that usually have one or more hydrogen atoms fewer than the ionic recombining species. Neutrals can also be produced via radiative association or neutral-neutral processes and can be destroyed by photodissociation. Interestingly, generation of poor-hydrogen containing species and insertion of carbon into molecules via reactions involving $\mathrm{C}^{+}$(ion-molecule) or $\mathrm{C}$ (neutral-neutral) lead to the production of long carbon chains. Concerning negative ions, they are expected to be mainly produced via simple radiative attachment of an electron to the parent neutral. Dissociative attachment is another mechanism which is efficient at low temperature only for weakly bound neutral reactants. Destruction of anions should occur via associative detachment, ion-molecule or mutual neutralisation reactions.

Concerning planetary atmospheres, this list of processes is also to be taken into account in addition to three-body reactions. Giant Planets chemistry is driven by hydrogen (main component of their atmosphere) whereas Titan has an atmosphere dominated by nitrogen with a significant concentration of methane and $\mathrm{CO}_{2}$ is the main component of Mars's atmosphere. Other specific problems of planetary 
Table 1. List of the main chemical processes of interest for astrophysical environments.

$\begin{array}{ll}\text { ion-molecule reactions } & \\ \text { Charge Transfer } & \mathrm{A}^{+}+\mathrm{B} \rightarrow \mathrm{B}^{+}+\mathrm{A} \\ \text { Radiative Transfer } & \mathrm{A}^{+}+\mathrm{B} \rightarrow \mathrm{AB}^{+}+\mathrm{h} v \\ \text { Atomic Transfer } & \mathrm{AB}^{+}+\mathrm{C} \rightarrow \mathrm{A}^{+}+\mathrm{BC}\end{array}$

Electron recombination reactions

Radiative

$\mathrm{A}^{+}+\mathrm{e} \rightarrow \mathrm{A}+\mathrm{h} v$

Dissociative

$\mathrm{AB}^{+}+\mathrm{e} \rightarrow \mathrm{A}+\mathrm{B}$

Photochemical processes

Photodissociation

$\mathrm{AB}^{(*)}+\mathrm{h} v \rightarrow \mathrm{A}+\mathrm{B}$

Photoionisation

$\mathrm{AB}+\mathrm{h} v \rightarrow \mathrm{AB}^{+}+\mathrm{e}$

Neutral neutral reactions

Atom or Molecule Transfer

Radiative Association

$\mathrm{A}+\mathrm{BC} \rightarrow \mathrm{AB}+\mathrm{C}$

$\mathrm{A}+\mathrm{B} \rightarrow \mathrm{AB}+\mathrm{h} v$

Other

$\begin{array}{ll}\text { Ion-Ion neutralisation } & \mathrm{A}^{+}+\mathrm{B}^{-} \rightarrow \mathrm{AB} \\ \text { Anion - Neutral Reactions } & \mathrm{A}^{-}+\mathrm{B} \rightarrow \mathrm{AB}+\mathrm{e} \\ & \mathrm{A}^{-}+\mathrm{BC} \rightarrow \mathrm{AB}^{-}+\mathrm{C} \\ \text { Electron Attachment } & \mathrm{A}+\mathrm{e} \rightarrow \mathrm{A}^{-} \text {or } \mathrm{AB}+\mathrm{e} \rightarrow \mathrm{A}^{-}+\mathrm{B} \\ \text { Three Body Reactions } & \mathrm{A}^{(* *)}+\mathrm{B}+\mathrm{M} \rightarrow \mathrm{AB}+\mathrm{M}\end{array}$

(*): here $\mathrm{AB}$ can be either an ion or a neutral molecule.

$(* *)$ : here A can be either an ion or a neutral species.

atmospheres are also taken into account into the photochemical models such as transport and eddy diffusion which have a significant role in the evolution of concentrations. Ion-molecule chemistry essentially occurs in the ionosphere, whereas neutral chemistry is dominant in the stratosphere although not totally absent in the upper atmosphere as well.

The following table summarizes the different families of reactions and photochemical processes that are of importance in astrophysical environments.

The aim of the present chapter will be to give an overview of the present laboratory experiments which can determine rate coefficients and branching ratios for chemical reactions, and more specifically at sub ambient temperatures. As the field is extremely wide, the objective of the present document will be to deliver key references to help the reader in going deeper into the topic. Some techniques will be briefly described with some focus on their strength, complementarities and limitations. Perspectives in this field will be presented eventually.

\section{EXPERIMENTAL TECHNIQUES FOR THE STUDY OF REACTION KINETICS AT VERY LOW TEMPERATURES.}

\subsection{General comments and aspects}

When a chemical network is built in order to study the evolution of the considered species, a series of equations of the following form are written:

$$
\frac{d[A]}{d t}=\sum_{j} k_{j}\left[B_{j}\right]\left[C_{j}\right]-\left(\sum_{i} k_{i}\left[D_{i}\right]\right)[A] .
$$


They describe the temporal evolution of the concentration of species A, taking into account production ( $j$ terms) and destruction ( $i$ terms) contributions. The $k_{j}$ and $k_{i}$ parameters are defined as the rate coefficients for every elementary process. They are expressed in units $\mathrm{of} \mathrm{cm}^{3}$ molecules $\mathrm{s}^{-1} \mathrm{~s}^{-1}$. Usually they are temperature dependent and for three-body processes they are also pressure (or concentration) dependent. As they result from the thermal average of a very large number of reactive collisions they represent a macroscopic quantity that can be measured experimentally. Cross sections $\sigma$, on the other hand, are microscopic quantities, expressed in $\mathrm{cm}^{2}$ units, which reveal the efficiency of a single collision in the presence of two partners with specific internal states and having a relative velocity $\mathrm{v}$ (i.e. a centre of mass translational energy $E_{T}$ ). Both quantities are related to each other by the standard equation:

$$
k(T)=\left(\frac{8 k_{\mathrm{B}} T}{\pi \mu}\right)^{1 / 2}\left(k_{\mathrm{B}} T\right)^{-2} \int_{0}^{\infty} \sigma\left(E_{T}\right) E_{T} \exp \left(-E_{T} / k_{\mathrm{B}} T\right) d E_{T}
$$

where $k_{B}$ and $\mu$ are the Boltzmann constant and the reduced mass respectively. Chemical reactivity can therefore be studied experimentally using several approaches. To obtain thermal averages, experiments are most often conducted in flow tubes or uniform supersonic flows at a density sufficient to ensure that the large number of collisions maintain local thermodynamic equilibrium (LTE). This is not the case for cross section measurements, which are performed with beams in the molecular free regime and with very few collision events. These are also very difficult measurements, since very low temperatures correspond to very low $E_{T}$. In order to obtain a rate coefficient by convolution following Eq. 2, such experiments need to be done with a sufficiently high resolution, especially at the lowest collision energies.

The nature of the reagent is another matter of concern. Historically the experimental techniques that have been developed in the field of very low temperature chemistry have first dealt with charged species $[18,19]$. The main reason is that ions can be easily manipulated, stored by electromagnetic fields and observed with high sensitivity, giving rise to a large variety of techniques which are not applicable to neutral chemistry. Most of these techniques were developed in the 1980s and early 1990s and have been reviewed elsewhere [20]. Collisions involving electrons deserve a special discussion since, due to their very low mass, $E_{T}$ is essentially the electron kinetic energy. It is rather easy, especially with laser techniques, to generate electrons of very low energies which do not relax efficiently to the higher neutral gas temperature. It is therefore quite simple to study cross sections at low $E_{T}$ for processes such as electron attachment [21] but these studies have been conducted mostly with internal temperature of the neutral molecule close to $300 \mathrm{~K}$.

Designing experiments in which very low temperatures can be achieved is absolutely mandatory although this has required high level technical developments. An approach that is often considered when no data are available at interstellar relevant temperatures is to extrapolate results obtained at temperatures around the usual $300 \mathrm{~K}$ conditions for which a large quantity of reactions have been studied (see for instance [22-24]) and temperature dependencies are sometimes provided in a limited range of temperatures. The expectation is that rate coefficients will follow a simple trend in their temperature evolution and therefore, extrapolation from say $300 \mathrm{~K}$ to $10 \mathrm{~K}$ should lead to an acceptable order of magnitude of the desired rate coefficient. We now know that this situation is far from being the usual behaviour of rate coefficients as illustrated in Fig. 1 for a series of neutral neutral reactions $\left(\mathrm{Al}+\mathrm{O}_{2}\right.$; $\mathrm{B}+\mathrm{C}_{2} \mathrm{H}_{2} ; \mathrm{CN}+\mathrm{C}_{2} \mathrm{H}_{6}$ and $\mathrm{CN}+\mathrm{CH}_{2}=\mathrm{C}=\mathrm{CH}_{2}$ ). For instance, extrapolating the data obtained for the reaction $\mathrm{CN}+\mathrm{C}_{2} \mathrm{H}_{6}$ in the temperature range $300-700 \mathrm{~K}$ to $25 \mathrm{~K}$ lead to a calculated value that is several orders of magnitude lower than the experimental measurement !

The more intuitive method to cool down a gas is based on cryogenic cooling. In this kind of experiment, a gas mixture (buffer, precursors and reagents) is introduced in a cell whose walls can be refrigerated (or heated) using different fluids. The gas flow can be almost static or can move at a subsonic speed. This technique, however, suffers from the disadvantage that any gas which is present above its saturation vapour pressure will condense on the walls of the cooled apparatus. One must then keep the reagents at partial pressures below their vapour pressure at the wall temperature. Nevertheless, 

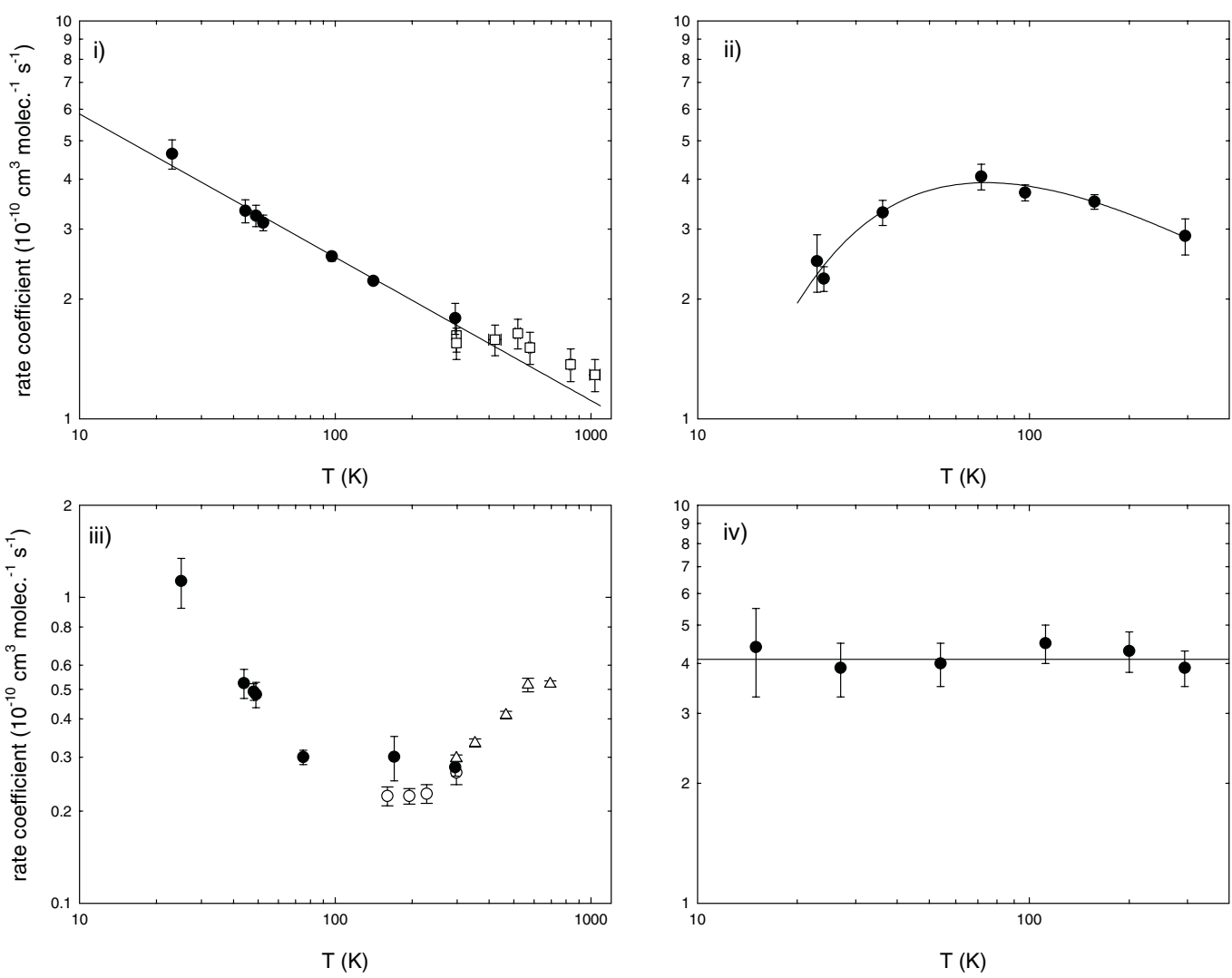

Figure 1. Rate coefficients for the reactions: i) $\mathrm{Al}+\mathrm{O}_{2}$, ii) $\mathrm{B}+\mathrm{C}_{2} \mathrm{H}_{2}$, iii) $\mathrm{CN}+\mathrm{C}_{2} \mathrm{H}_{6}$ and iv) $\mathrm{CN}+\mathrm{CH}_{2}=\mathrm{C}=$ $\mathrm{CH}_{2}$.

the concentration must be high enough to perform kinetics experiments and only gases with a significant vapour pressure at the wall temperature are then suitable. These kinds of devices are perfectly suited for studies of atmospheric interest [25] for which the minimum temperature is about $180 \mathrm{~K}$. By using liquid nitrogen as a coolant however, some experiments have been carried out on reactive processes down to $80 \mathrm{~K}[26,27]$.

Another kind of techniques which does not suffer from the condensation problem is based on the supersonic expansion of a non condensable buffer. Here, cooling arises from conservation of energy which implies that for a gas flow under adiabatic conditions, the sum of specific enthalpy and kinetic energy remains constant during the expansion. For a perfect gas with a constant specific heat capacity $\mathrm{c}_{p}$ and with a well defined temperature, the energy conversion is driven by the following very simple equation:

$$
c_{p} T+\frac{u^{2}}{2}=c_{p} T_{0}
$$

where $T_{0}$ and $T$ are the temperatures of the gas prior and after expansion respectively and $u$ is the supersonic flow velocity.

The simplest type of supersonic expansion is the so-called free jet expansion, in which a gas at high pressure escapes through a small orifice into a high vacuum chamber. Here the cooling can be extreme: down to $c a .0 .1 \mathrm{~K}$. In this case the medium is inhomogeneous and the thermodynamic conditions are extremely complex. As the expansion evolves, the collision frequency decreases and therefore 
the different temperature moments (translation, rotation and vibration) cannot reach equilibrium [28] making it difficult to assign a "true" thermodynamic temperature. The strong gradients of temperature and density, the low frequency of collisions and the short useable area of the jet have prevented its generalised use in studies of chemical reactions although several ion-molecule reactions have been investigated [20]. For these processes, the long range electrostatic forces are strong enough to attract together reagents travelling with very similar translational velocity.

The insertion of a "skimmer" into the supersonic zone of the free jet generates a molecular beam [29] with a well defined velocity. By making an interaction between two molecular beams, collisions can be studied at a well defined relative translational energy. This is the basis of the so-called crossed beams method with which reactive cross sections can be measured. Usually beams collide at right angle although some apparatus have been designed with the possibility of varying the collision angle [30, 31]. Low $E_{T}$ can be achieved with this technique when the angle is very small or/and when one partner is light such as $\mathrm{H}_{2}$. The merged beams technique [32-34] is a specific crossed beams device in which the collision angle is zero. In this case extremely low relative energies can be achieved. Whereas the standard crossed beams can be used for the study of both neutral-neutral and ion-molecule reactions, the merged beams technique is only applicable for investigations involving charged species because it is not technically feasible to merge two neutral beams at zero angle.

Finally, a low temperature chemical reactor can be obtained using "collimated" uniform supersonic flows generated by the isentropic expansion of a buffer gas introduced in a reservoir and passing through an axisymmetric convergent-divergent Laval nozzle. This is the so-called CRESU technique (Cinétique de Réaction en Ecoulement Supersonic Uniforme / Reaction Kinetics in a Uniform Supersonic Flow) for which the temperature is a "true" temperature, i.e. LTE prevails in the flow. Uniform supersonic flows were initially developed in a continuous flow version for the study of ion-molecule reactions [35]. Later, the methodology was adapted to the study of reactions between neutral species in a continuous flow version [36-38] and also in a pulsed flow version [39-43].

In Figure 2, the three kinds of supersonic flows described in the preceding paragraphs are schematically represented.

\subsection{Overview of experimental techniques}

\subsubsection{Ion-molecule reactions}

The development of mass spectrometry along the last five decades has been a decisive step for the building up of a series of experiments dedicated to the study of reaction kinetics of charged species [44]. These include instruments such as the ion cyclotron resonance (ICR) cell; flow tubes (flowing afterglow (FA), flowing afterglow-Langmuir probe (FALP), flowing afterglow with photoions FlAPI), selected ion flow tube (SIFT)); Fourier Transform Mass Spectrometry (FTMS) apparatus; ion beams, traps and storage rings; and supersonic expansions devices (free jet, CRESU). Only a limited number of these powerful tools, however, were able to deliver data at the lowest temperatures prevailing in the coldest environment of the interstellar medium. It is in the 1980s and early 1990s that several techniques were developed for the study of ion-molecule reactions down to temperatures close to $10 \mathrm{~K}[20,45]$.

A first group of methods makes use of cryogenic cooling which is not a problem for the ionic partner of the reaction, since ions can be maintained far from the walls using suitable fields, therefore inhibiting their heterogeneous condensation. At the lowest temperatures, however, these techniques are usually restricted to co-reagents of high vapour pressure, especially molecular hydrogen and its deuterated forms. Probably one of the presently most popular technique is the SIFT apparatus [46]. This is a standard flow tube experiment in which ions are produced separately by electron impact. The generated ions (cations or anions) are then mass-filtered using a quadrupole mass spectrometer so that only the desired charged species is introduced in the main flow containing a buffer gas and the reagent of interest. Although the technique is largely spread over the world now, most of the data 


\section{Chemistry in Astrophysical environments}
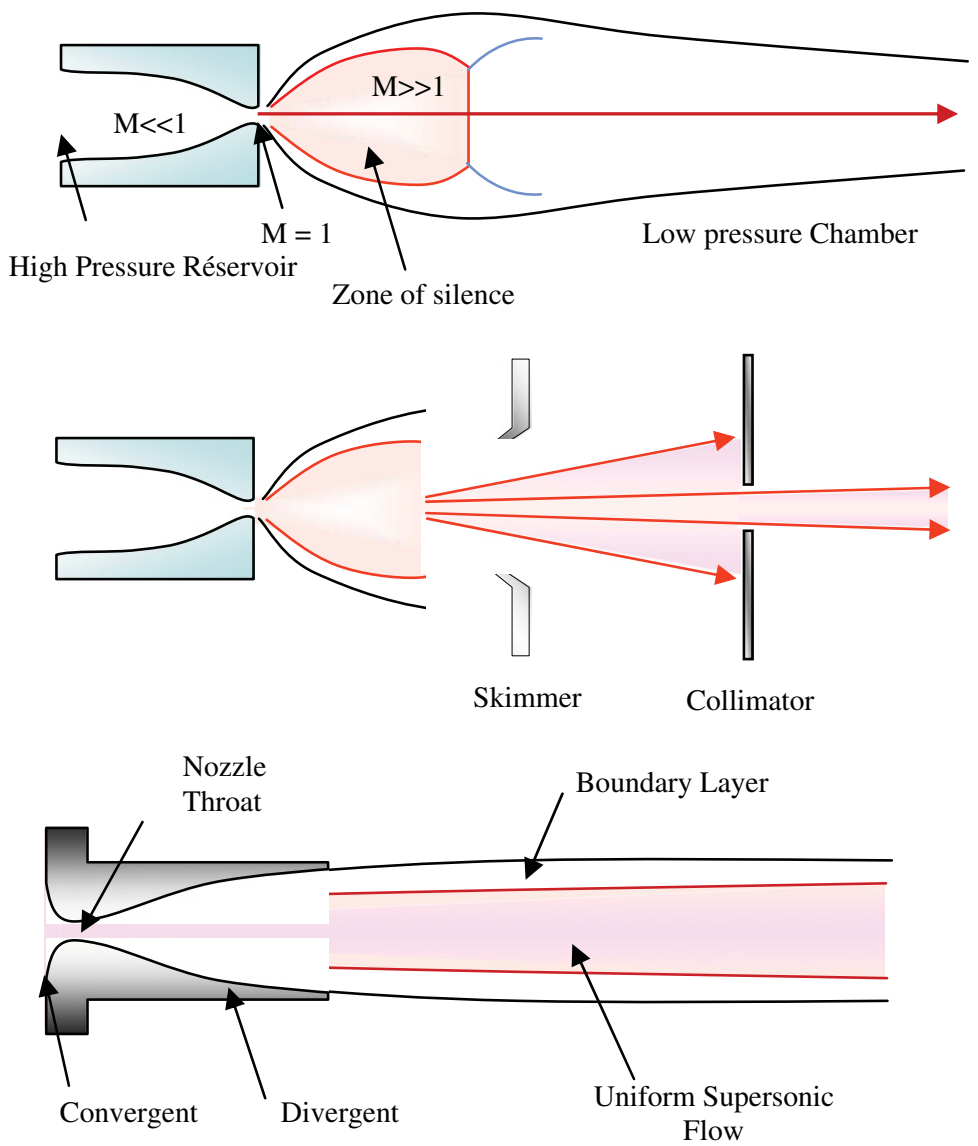

Figure 2. Schematic views of three kinds of supersonic expansions.

are obtained at room temperature and the lowest achievable temperature remains about $80 \mathrm{~K}$. The very first measurements on ion-molecule reactions at temperatures below $80 \mathrm{~K}$ were reported in 1983 [47]. Using a drift tube cryogenically cooled by liquid helium, temperatures as low as $20 \mathrm{~K}$ were routinely obtained. However, in this technique the ions are not strictly equilibrated at the apparatus temperature and their velocity distribution is not Maxwell-Boltzmann because of the presence of electric fields [20]. This method, as well as the cryogenically cooled Penning trap experiment of Dunn and coworkers [48], has not been widely used and most of the developments of cryogenically cooled methods have been performed by Gerlich and co-workers using radiofrequency traps with special geometries to minimise heating of the ions due to radiofrequency fields [45, 49]. It is worth mentioning a series of recent experiments on ion $-\mathrm{H}$ reactions using an innovative version of the apparatus [49, 50]. Here rate coefficients have been obtained for $\mathrm{CH}^{+}, \mathrm{CH}_{4}^{+}, \mathrm{CH}_{5}^{+}$and $\mathrm{CO}_{2}^{+}$ions for an ion temperature ranging from 10 to $300 \mathrm{~K}$. Interestingly, the interaction time in ion traps can be varied typically from $\mu \mathrm{s}$ to min or longer and the number density of neutrals can also be varied from below $10^{9} \mathrm{~cm}^{-3}$ to above $10^{14} \mathrm{~cm}^{-3}$. Therefore by choosing a suitable combination of these two parameters, the rate coefficient of rather fast as well as rather slow bimolecular reactions can be measured. Beyond the study of ion molecule reactions at temperatures as low as $10 \mathrm{~K}$, due to the long residence time of the 


\section{EPJ Web of Conferences}

confined ions, traps were also used to investigate the usually slow radiative association process [51, 52] which is of great interest for the interstellar medium because of the low densities reigning in these environments.

The second group of methods relies on supersonic expansion. In the early 1980s the group of Rowe, developed the CRESU technique: an ion-molecule reactor that made use of the uniform supersonic flows generated continuously by a Laval nozzle [35]. In this experiment, ions were generated by electron impact ionisation of the buffer gas ( $\mathrm{He}, \mathrm{N}_{2}$ or $\mathrm{Ar}$ ) and/or a suitable parent gas making use of a high energy electron beam crossing the supersonic flow perpendicularly. Evolution of the ion concentrations were probed by means of a moveable mass spectrometer. The first measurements of rate coefficients down to $20 \mathrm{~K}$ were reported in 1984 and soon results for temperatures as low as $8 \mathrm{~K}$ were obtained $[18,19]$. In an attempt to measure exact branching ratios of reactions a selective ion injection source was added to this apparatus in 1988 [53]. It made use of a quadrupole filter to select reagent ions that were injected in the nozzle boundary layer and subsequently drifted into the core of the flow by suitable electric fields. The great advantage of this source was that the neutral parent of the reagent ion was not present in the flow thus avoiding an additional destruction route of the ion. In 1993 part of this apparatus was moved to the University of Rennes 1 where it was rebuilt and adapted to the use of slightly higher pressures. In addition, a pulsed ion source [54] was further developed, allowing the injection of ions into the flow by drifting them in electric fields and avoiding the presence of parent neutrals, as well as a variety of excited species initially present in the flow with the electron beam technique. By pulsing the drifting field, ions penetrated the core of the flow where they could cool down to the flow temperature prior to reaction, thereby avoiding the field heating problem common in drift techniques [47, 54].

Following closely the work of Rowe and co-workers, M.A. Smith and co-workers have developed an ion-molecule reactor based on the free jet expansion $[55,56]$. To avoid very large pumping capacities and high gas consumption the jet was operated in a pulsed mode at $10 \mathrm{~Hz}$. Ions were created by laser multiphoton ionisation of a suitable precursor, ten to thirty nozzle diameters (typically ranging from 0.1 to $1 \mathrm{~mm}$ ) downstream of the nozzle exit plane, where the velocity has reached a value very close to the hypersonic limit ( $\mathrm{T} \equiv 0$ in Eq. 3). From this point the flow continues to expand at all downstream positions and ions eventually react with neutral molecules. Reagent and product ions are monitored versus distance to the laser beams using a time of flight mass spectrometer set at right angles to the flow. To extract the rate coefficient, it was necessary to take into account that density continuously drops along the flow axis. Therefore, in the ion reaction zone the neutral-neutral collision rate is negligible, causing strong thermodynamic disequilibrium within the jet. The velocity distribution is no longer MaxwellBoltzmann but it was shown that it remains possible to assign a well-defined kinetic average temperature for the bulk rate coefficient measured [20]. More problematic is the question of rotational temperature which can diverge quite far from the kinetic temperature [56] and cannot always be modelled due to the lack of data on rotational relaxation in this energy range. Even if the temperature and the thermodynamic conditions cannot be assigned as well as in a CRESU experiment, the free jet reactor has been able to produce data for some reactions at an equivalent kinetic temperature of $0.3 \mathrm{~K}$ which is still an ultimate performance in this field [20]. However it has proven impossible to extend the technique to neutralneutral reactions.

The merged beam experiment of Gerlich and co-workers [33] can be considered as belonging to both groups of techniques: skimmed supersonic expansion is used here to produce a dense beam of neutrals with cooled controlled internal states. It is merged with an intense slow guided ion beam which makes extensive use of cryogenically cooled traps for ion production and relaxation. By matching the ion beam velocity to the hypersonic terminal velocity of the neutral beam, extremely low $E_{T}$ have been obtained $(\sim 1 \mathrm{meV}$ i.e. $\sim 10 \mathrm{~K})$. With the molecular beam and ion guiding techniques available today, sub-meV $(<10 \mathrm{~K})$ collision energies are potentially achievable [45]. 


\subsubsection{Electron Attachment}

This process is somewhat special because its efficiency may depend both on the electron and the neutral temperature that can be different from each other in experiments and natural environments. Techniques allowing electron attachment to be studied at very low electron energy have been described in several reviews (see for example [21]). They can be separated into two categories: single collision, and multiple collision techniques.

In the former class, the electrons are produced at very low energy and collide with the molecules. The latter class includes LTE techniques which produce electrons that are injected into a buffer gas and are thermalised by collisions with this gas.

In experiments performed under single collision conditions, the use of Rydberg atoms and threshold photoionisation techniques of rare gases are the two most representative techniques for producing electrons. The former experiment does not use low energy electrons but instead uses the weakly bonded electron of a high Rydberg level of an atom (see for example [57, 58]). High Rydberg states of atoms, such as xenon or rubidium, are generated by laser irradiation and react directly with molecules via electron transfer. Due to the weak bonding of the electron, the reaction rate of the Rydberg atom with the molecules is identical to the attachment rate of electrons with the molecules, assuming that the electrons have the same velocity distribution as the reacting Rydberg atoms. The number density of the Rydberg atoms is followed in time by a selective ionising field. Such techniques allow measurement within an energy range from $0.05 \mathrm{meV}$ to $40 \mathrm{meV}$.

On the other hand, photoionisation techniques produce low energy free electrons and measure the direct attachment rate [21]. Electrons are generated either by direct near threshold photoionisation of a gas using VUV light or by exciting argon atoms by electron impact, then using a two photon process to generate argon ions and threshold electrons. In both cases the energy of the resulting electrons is the difference between the total energy provided to ionise the atom and its ionisation energy. After reactions with the low energy electrons, the negative ions formed are detected by mass spectrometry. Better energy resolution is obtained using the multi-step process.

The FALP technique is characteristic of experiments performed at LTE. This technique was developed by D. Smith, N.G. Adams and co-workers to study the reactivity of charged species including electron-molecule collisions down to $200 \mathrm{~K}[59,60]$. In the FALP approach an afterglow plasma is generated in a fast-flowing helium carrier gas by means of an upstream microwave discharge. Argon atoms are added to the plasma to quench any metastable helium atoms, and a small quantity of the target gas is added downstream. At the point of this addition a large axial gradient of the electron density is generated due to continual depletion by attachment to the molecule. Measurement of the attachment rate coefficient is achieved by measuring the electron density along the axis of the flow, using a Langmuir probe, for different molecular densities.

Other techniques are available to study electron attachment. However, most of them do not permit one to vary the temperature of the molecular species from room temperature to low temperature in true LTE. For this purpose, the CRESU apparatus may be the most suitable technique as neutrals are naturally cooled down very efficiently due to the large number of collisions with the buffer gas during the flow expansion. Several studies have been carried out with this apparatus in which an electron beam creates a plasma with a moderate level of ionisation $\left(10^{8}\right.$ to $\left.10^{9} \mathrm{e}^{-} \mathrm{cm}^{-3}\right)$. The electron density can be monitored easily by a Langmuir probe and the produced anions by a moveable mass spectrometer. Nitrogen was known to be very efficient at relaxing electrons towards the flow temperature [61] and was therefore used as the buffer. However, careful studies [62] have shown that, in this experiment, the electrons relax to a temperature higher than that in the flow. It is worth mentioning here, that in astrophysical environments the electron temperature is not necessarily equilibrated to that of the neutrals. For instance, in Titan's ionosphere, the electron temperature can be greater than $1000 \mathrm{~K}$ [63] whereas the temperature of the neutral is typically $150 \mathrm{~K}$. In the interstellar medium the situation is not completely clear as well. 


\section{EPJ Web of Conferences}

\subsubsection{Dissociative Recombination}

The dissociative recombination (DR hereafter) of a polyatomic cation with an electron is one of the most complex molecular collision processes and it has been considered being an enormous challenge to researchers for about sixty years. It is one of the main providers of neutral species in interstellar space and therefore it plays a central role in astrochemistry. For example, the rate coefficient for the dissociative recombination of the $\mathrm{H}_{3}^{+}$ion is critical in the chemical scheme of interstellar clouds. If this rate is low enough then $\mathrm{H}_{3}^{+}$can react with neutrals and initiate an ion chemistry which results in generating more and more complex molecules.

Therefore, the experimental work that has been undertaken concerning this kind of reaction is very abundant and two recent review papers are of great help to get a synthetic view of what is presently available [24, 64]. Historically the field was dominated by two classes of instruments: flow tubes and crossed (merged) beams.

The FALP apparatus developed at the University of Birmingham (GB) by D. Smith and N.G. Adams rapidly became a reference device in the early 1980s for the measurement of DR rate coefficients at temperatures ranging from $100 \mathrm{~K}$ to $600 \mathrm{~K}[65,66]$. In this flow tube technique, a microwave discharge produced a plasma within a buffer gas operating typically at 1 mbar. The many collisions occurring in this flow ensures thermalisation of ions and electrons to the neutral gas, the temperature of which could be varied cryogenically. A moveable Langmuir Probe was used to follow the spatial electron density whereas a mass spectrometer permitted identification of ions present in the flow. Initially, these experiments were only dedicated to the measurement of the rate coefficient at sometimes variable temperatures. The lowest temperature achievable using the FALP technique is $80 \mathrm{~K}$ given by the liquid nitrogen cooling of the walls of the apparatus [67].

The necessary knowledge of the nature of exit channels and more fundamentally the determination of the associated branching ratios led to the development of new techniques to measure the concentration of neutral products in the flow. Pioneering work in this field (at least for thermal electrons) was undertaken in a coordinated collaborative programme in Birmingham and Rennes (France) and led to the detection of $\mathrm{OH}$ radicals using Laser Induced Fluorescence (LIF) and hydrogen atoms by VUV absorption $[68,69]$. Spectroscopic techniques have been used as well at the University of Pittsburg (USA) by the group of R. Johnsen to observe the production of $\mathrm{CO}, \mathrm{N}_{2}, \mathrm{OH}$ and $\mathrm{CN}$ molecules in the DR of $\mathrm{CO}_{2}^{+}$ [70, 71], $\mathrm{N}_{2} \mathrm{H}^{+}$[72] $\mathrm{H}_{3} \mathrm{O}^{+}[73,74]$ and $\mathrm{HNC}^{+}$[75] respectively. Very recently, the FALP apparatus available at the University of Georgia (USA) has been adapted for the study of neutral products issued from the DR process $[76,77]$. The main upgrade was to use an electron impact ionizer to identify the neutrals from the detection of the associated cations by mass spectrometry. Pulsed gas flow techniques were also implemented for the measurement of branching ratios. The technique has presently been applied to $\mathrm{N}_{2} \mathrm{H}^{+}$and $\mathrm{CH}_{5}^{+}$.

The mid 1990s, saw the first application of heavy-ion storage rings to the study of the DR process $[34,78]$. The original idea of this new development was the successful adaptation and amplification of the merged beam technique of McGowan and co-workers [79] that in turn had greatly broadened the field of study in the 1980s. Several ion storage ring equipments over the world have been partly dedicated to the study of dissociative recombination. These are in Stockholm (CRYRING, Sweden), Aarhus (ASTRID, Denmark), Heidelberg (TSR, Germany) and Tokyo (TARN II, Japan). Ion production is made inside an ion source through electron impact on a parent gas. They are accelerated to several tens of $\mathrm{keV}$ and mass analysed before being injected into the synchrotron where they are accelerated to several $\mathrm{MeV}$ and then stored so that they circulate in the magnetic lattice of the ring for a period of several seconds to several tens of seconds. During each revolution, the ion beam merged with an intense electron beam along an interaction zone of typically $1 \mathrm{~m}$ in length. The velocity of the electrons can be made to match that of the ions or it can be varied so that collisions can be studied over a range of centre-of-mass collision energies. Neutrals formed in the DR reactions have a straight trajectory after the interaction zone and are counted outside the ring by a detector. 
This can be an energy sensitive surface-barrier detector used for rate coefficient and branching ratios measurements or a time and position sensitive 3D detector for studies of energy distribution into final channels.

In this kind of experiments, for a given collision energy, what is effectively measured is a rate coefficient which is a mean cross section $\langle\sigma v\rangle$ over the energy resolution of the collision. The measured rate is then expressed in the form:

$$
\langle\sigma v\rangle=\int v \sigma(v) f\left(v, v_{r e l}\right) d v .
$$

Therefore, to obtain the true cross section, one needs to know the velocity distribution of the electrons in the centre of mass frame $f\left(v, v_{r e l}\right)$ and invert Eq. 4 using Fourier Transform techniques. Once the cross section $\sigma(v)$ has been calculated from this method and that experiments have been carried out over a sufficiently large collision energy range, the rate coefficient can be obtained from Eq. 2 as a function of the electron temperature. As very low collision energies can be achieved $(\sim 1 \mathrm{meV})$ the temperature dependence of the rate coefficient can be reliable down to interstellar space conditions and it is usually given in the form:

$$
\alpha(T)=\alpha(300 K)\left(\frac{300}{T}\right)^{n} .
$$

The use of storage rings for the study of DR led to a considerable breakthrough which produced a cascade of new data and above all crucial information about the temperature dependence of the rate coefficient. For a synthetic view of the whole work that has been accomplished in this field, we refer the interested reader to the review paper by A. Florescu-Mitchell and J.B.A. Mitchell [24].

However, and beyond this considerable progress, the major strength of storage rings was their ability to determine branching ratios directly by separating out the different dissociation energy channels of the neutrals produced in the DR process taking advantage of the energy resolving characteristics of a surface barrier detector. Very briefly, a mesh grid, with a known transmission T, was placed in front of the detector so that the probability for one neutral to reach the detector is $\mathrm{T}$, for two neutrals it is $\mathrm{T}^{2}$ etc..Thus, when not all neutrals issued from a recombination event arrive at the detector, this will perceive a lower energy than if all particles had reached it. Once all possible channels have been identified, it is then possible to write a series of equations taking into account the desired branching ratios as well as the corresponding probabilities of detection (all this terms being products of the form $\left.\mathrm{T}^{n}(1-\mathrm{T})^{\mathrm{m}}\right)$ to calculate the number particles corresponding to every energy level observed by the surface detector. From this, branching ratios can be extracted. Further details of the technique can be found in $[80,81]$. Here again, the number of resulting data from this method is considerable and a compilation can be found in [24]. Note that the data for $\mathrm{H}_{3} \mathrm{O}^{+}$are erroneous in this compilation for the CRYRING measurements and therefore we recommend looking at the original work [82]. One of the more striking result that was obtained by these experiments concerned hydrocarbon ions for which a large number of different product channels have been observed (including those involving carbon-carbon breaking bounds) and the dominance of a particular channel appears in several cases to be determined by the structure of the initial recombining ion [83-86].

\subsubsection{Neutral-neutral reactions}

For many years most neutral-neutral reactions were expected to be slow at the very low temperature of dense clouds as they were thought to have positive activation energies $E_{a}$. If the temperature dependence of the rate coefficient $k$ is described by the Arrhenius equation $\left(k(T)=A \exp \left(-E_{a} / R T\right)\right)$ then the rate coefficients at $c a$. $10 \mathrm{~K}$ will be insignificantly small. Only a few atom-radical reactions such as $\mathrm{O}+\mathrm{OH} \rightarrow \mathrm{O}_{2}+\mathrm{H}, \mathrm{O}+\mathrm{CH} \rightarrow \mathrm{CO}+\mathrm{H}, \mathrm{N}+\mathrm{OH} \rightarrow \mathrm{NO}+\mathrm{H}$ etc... were considered in models because this class of processes was expected to proceed without any barrier [87]. In the early 1990s 
experimental developments shed new light on radical-neutral reactions and demonstrated that a large number of these processes, even those involving a saturated molecular partner, can be very efficient at temperatures as low as $13 \mathrm{~K}$ [18]. This discovery was carefully considered by ISC modellers who analysed the impact of these new and revolutionary observations [6]. The number of neutral-neutral processes included in models was significantly increased and rate coefficients were re-evaluated on the basis of the new experimental data. The main consequence of this upgrading was to reduce the rate of formation of complex species as a result of the prompter destruction of small radicals which initiate the chemistry leading to complex molecules.

In planetary atmospheres the problem was different for several reasons: the photochemistry in the stratospheres is essentially neutral, the temperatures are quite higher than in dense clouds and termolecular reactions must be considered as well due to significantly higher pressures in these environments. Excepting Pluto and Triton, the minimum temperature found in planetary atmospheres and their moons is always greater than $70 \mathrm{~K}$ so that the need for low temperature data is more limited than in interstellar space.

Reactions between neutral species have been studied experimentally for several decades mainly because of their atmospheric interest. As the temperature range for this environment is limited to 180$300 \mathrm{~K}$, cryogenic cells were usually best suited for this kind of investigations [25]. For temperatures lower than $80 \mathrm{~K}$, to date, the CRESU technique has no equivalent for the exploration of chemical reactivity in the gas phase. It is worth mentioning that even for atmospheric conditions, the CRESU apparatus can be more appropriate than a cryocell when the studied reagents are highly condensable or even are liquids or powders at the laboratory temperature. As a matter of example, the reactivity of anthracene (a PAH made of three linearly structured benzene cycles) which is a powder at room temperature, was studied in the temperature range $58-470 \mathrm{~K}$ in the presence of $\mathrm{OH}$ and $\mathrm{CH}$ radicals $[88,89]$, by means of the CRESU apparatus because the uniform and supersonic nature of the flow avoids condensation. On the other hand, due to the supersonic nature of the CRESU flow, only fast rate coefficients can be measured (typically $>5 \times 10^{-13} \mathrm{~cm}^{3}$ molecule $\mathrm{s}^{-1} \mathrm{~s}^{-1}$ ). This is not a problem with cryogenic cells which are often well suited for the study of slow reactions (typ. $k \sim 10^{-15}$ $10^{-13} \mathrm{~cm}^{3}$ molecule $\mathrm{s}^{-1} \mathrm{~s}^{-1}$ ) because reaction times of several milliseconds can commonly be achieved and relatively high concentrations of reagents can be introduced into the cell. Both techniques are therefore quite complementary.

As previously indicated, the CRESU apparatus was originally designed for the study of ion molecule reactions. In the early 1990s, however, in a coordinated collaborative project involving the University of Rennes (France) and the University of Birmingham (GB); B.R. Rowe, I.W.M. Smith and their coworkers adapted this powerful chemical reactor to the study of neutral - neutral reactions by coupling the continuous uniform supersonic flow to laser techniques. The so-called and largely spread PLP-LIF (Pulsed Laser Photolysis - Laser Induced Fluorescence) technique was implemented in the CRESU experiment in Rennes for this purpose. This method makes use of two pulsed lasers which are combined and co-propagate along the axis of the supersonic flow. The first laser produces an homogeneous concentration of the desired radical by photolysis of a suitable precursor which is introduced in the reservoir in addition to the buffer gas and the reagent of interest. Then, after a variable and controlled time delay, some of the radicals are excited to an upper electronic state by a tuneable laser acting as a probe. Fluorescence is collected at a known distance downstream of the Laval nozzle exit by means of an optically fast telescope-mirror combination mounted inside the main vacuum chamber at right angles to the supersonic flow and directed onto the photocathode of a (V)UV-sensitive photomultiplier tube after passing through an interference filter. For a given concentration of the reagent, the rate of loss $k_{1 s t}$ of the radical is observed by scanning the delay time between the two laser pulses using a delay generator. As the delay time increases the fluorescence signal decreases exponentially because radicals have been consumed in the reaction during their transit along the flow. In order to extract the required bimolecular rate coefficient $k, k_{1 s t}$ is measured as a function of the absolute molecular reagent concentration $[\mathrm{M}]$. Plotting this rate as a function of $[\mathrm{M}]$ leads to a straight line whose slope gives 
the required second-order rate coefficient $k$. More details concerning the CRESU experiment can be found in [18] as well as a compilation of results obtained until 2007. It is worth stressing, however, that this kind of study only probes the destruction of the reagent radical; hence no information can be extracted about the nature of the products and the branching ratios when several exit channels are open.

First results were obtained in 1992 for the $\mathrm{CN}+\mathrm{O}_{2}$ reaction which was studied in the temperature range $13-300 \mathrm{~K}[36,90]$. Since then, more than 80 neutral - neutral processes have been studied at very low temperature in Rennes, Birmingham (from 1996 to 2003) and more recently in Bordeaux [38] using a continuous version of the CRESU method. These include bimolecular reactions, three-body pressure dependent processes and also studies of energy transfer such as spin-orbit, rotational, vibrational and electronic relaxation.

In the mid 1990s a pulsed version of the CRESU technique was first developed by M.A. Smith (University of Arizona, USA) and co-workers [39]. Later, a similar apparatus was constructed in the group of S. Leone at Berkeley (USA) [40]. This has been mostly used to study reactions of the $\mathrm{C}_{2} \mathrm{H}$ radical with a wide range of hydrocarbons for Titan chemistry. More recently, in Troe's group [41], a pulsed Laval nozzle has been used to study the reactivity of the $\mathrm{OH}$ radical with important atmospheric compounds. Finally, in 2008, first results were published from the group in Leeds (GB) concerning the $\mathrm{OH}$ radical kinetics in the presence of unsaturated hydrocarbons [42]. In all these experiments laser techniques are employed, in the same way as in experiments using the continuous flow CRESU apparatuses.

The minimum temperature, however, is limited to $53 \mathrm{~K}$ [91, 92] due to quite small pumping capacities. This record for a pulsed apparatus was recently beaten in Rennes with the development of a new apparatus [43]. A pulsed supersonic flow was produced at $23 \mathrm{~K}$ using slightly higher pumping capacities and an innovative design for the pulsing system of the gas. Instead of using pulsed valves set at the entry of a small reservoir $\left(\sim a\right.$ few $\left.\mathrm{cm}^{3}\right)$ upstream of the Laval nozzle, as it is the case in previous pulsed devices, in Rennes the pressure in the 20 liter reservoir is maintained permanently very close to the working continuous conditions by chopping the gas in the divergent part of the Laval nozzle by means of a rotating disk. This has the main advantage to avoid periodic filling up and emptying of the reservoir thus ensuring that the gas inside the reservoir is almost stagnant. This is a crucial requirement for the validity of Eq. 3 which is the keystone for the determination of the supersonic flow temperature. Kinetic results have been obtained for two reactions at $23 \mathrm{~K}: \mathrm{CN}+\mathrm{O}_{2}$ and $\mathrm{CN}+\mathrm{C}_{3} \mathrm{H}_{8}$ and were found to be in perfect agreement with previous measurements carried out using the continuous CRESU.

For a long time it was quite difficult to rationalize the different studies in the field of neutral neutral reactions. The interpretation of the obtained results had to be done on a case by case manner. A few years ago, a survey of the existing measurements on very low temperature reactivity had been carried out by I.W.M. Smith an co-workers [93] who have proposed two methods for estimating low temperature reactivity. The first method, purely empirical, states that any radical-molecule reaction is likely to be rapid down to $10-20 \mathrm{~K}$ if its room temperature rate coefficient, $k(298 \mathrm{~K})$, is greater than ca. $5 \times 10^{-12} \mathrm{~cm}^{3}$ molecule ${ }^{-1} \mathrm{~s}^{-1}$, and if it has an activation energy $E_{\text {act }}$ at $298 \mathrm{~K}$ and above which is zero or negative. Conversely, reactions with $k(298 \mathrm{~K})<5 \times 10^{-12} \mathrm{~cm}^{3}$ molecule $^{-1} \mathrm{~s}^{-1}$ and $E_{\text {act }}>0$ are likely to become too slow at $10-20 \mathrm{~K}$ to be of importance in interstellar chemistry. This 'rule of thumb' appears to work well for the majority of low temperature reactions studied to date. However, there are some notable exceptions such as the strange behaviour of the $\mathrm{CN}+\mathrm{C}_{2} \mathrm{H}_{6}$ reaction [94] (see Fig. 1(iii)). This reaction has a relatively rapid room temperature rate coefficient $\left(\sim 3 \times 10^{-11} \mathrm{~cm}^{3}\right.$ molecule $\left.\mathrm{s}^{-1}\right)$, greater than $5 \times 10^{-12} \mathrm{~cm}^{3}$ molecule $\mathrm{s}^{-1}$, but displays a positive activation energy of about $2 \mathrm{~kJ} \mathrm{~mol}^{-1}$ at and above room temperature. However, low temperature measurements in the CRESU apparatus showed that its rate coefficient passes through a minimum, rising to its fastest measured value of $1.1 \times 10^{-10} \mathrm{~cm}^{3}$ molecule $\mathrm{s}^{-1}$ at $25 \mathrm{~K}$. It is now generally agreed that this behaviour can be understood in terms of a two transition state model (inner and outer) developed by S. Klippenstein 
and co-workers and which is found to be valid for several reactive processes (see [95-97] for details).

Following a work from Donahue and co-workers who have demonstrated that not only does the inner barrier in radical-molecule reactions arise from an avoided curve crossing between reagent ionic and covalent states, but that both the energy and other transition state properties are strongly correlated to reagent ionic energy [98], I.W.M. Smith et al. proposed a second, semi-empirical method for deciding whether rate coefficients would be substantial at very low temperatures [93]. When the difference between the ionisation energy of the molecular reagent (I.E.) and the electron affinity of the radical (E.A.), (I.E. - E.A.), is significantly greater than $8.75 \mathrm{eV}$, the reaction is likely to possess a 'true' barrier and will therefore become negligibly slow at $20 \mathrm{~K}$. On the other hand, reactions with (I.E. - E.A.) $<\sim 8.75 \mathrm{eV}$ are likely to be characterised by, at most, inner barriers that are submerged below the asymptotic reagent energy, and therefore be rapid at $20 \mathrm{~K}$. This assumption was successfully verified by the study of the reactivity of ground state oxygen atoms, $\mathrm{O}\left({ }^{3} \mathrm{P}\right)$, and simple alkenes possessing values of (I.E. - E.A.) that bridge the 'critical' value of $8.75 \mathrm{eV}$ [97]. It also held for a series of reactions involving the $\mathrm{C}_{4} \mathrm{H}$ radical and a series of hydrocarbons [99-101] which were investigated later. It is then an interesting criterion to consider, especially for reactions that are not easy to investigate experimentally at very low temperatures.

Another kind of method, that brings precious information on neutral neutral reactions, takes advantage of the combination of molecular beams. These have been used for a long time to obtain information on the mechanisms driving such processes and are very useful in studies of collisional dynamics. More particularly, the technique provides valuable data with respect to the nature of the products of the reaction and its mechanism [31]. As the frequency of collisions is very small in a molecular beam, data are obtained from experiments in which two collimated reagent beams intersect at a fixed angle (quite often $90^{\circ}$ ) in a scattering region where single collisions occur between the components of the beams. The species of each beam are characterised by a well-defined velocity and usually also internal quantum states. By varying the velocity of the molecular beams and their intersection angle, differential cross sections (product angular and velocity distributions) can be measured at different collision energies.

In most crossed molecular beam machines the usual collisional energies are rather high with respect to those encountered in the molecular rich regions of the interstellar space. However some devices are designed so that the beam intersection angle can be varied enabling the relative collision energy to be reduced. The lowest collisional energy presently available was obtained by C. Naulin and M. Costes [30] who were able to perform experiments using an apparatus for which the intersection angle can be varied from $90^{\circ}$ down to $22^{\circ}$, the latter corresponding to a collision energy of $0.35 \mathrm{~kJ} / \mathrm{mol}$ (i.e. $\sim 30 \mathrm{~K}$ ). Very recently, this record has been broken and a minimal collision energy of $0.066 \mathrm{~kJ} / \mathrm{mol}$ (i.e. $\sim 5 \mathrm{~K}$ ) was achieved for the study of collisions of metastable sulphur atoms $\mathrm{S}\left({ }^{1} \mathrm{D}\right)$ with molecular hydrogen [102]. In this experiment, the use of cryocooled pulsed valves to generate the beams allowed to slow down the colliding particles and the light $\mathrm{H}_{2}$ molecule favoured lower collision energies as well.

In crossed beam devices, the relative total cross sections (also referred as the excitation function) can be integrated assuming a Maxwell distribution of relative velocities at temperature $T$ to yield an estimate of the thermal rate coefficient, $k(T)$. This, however, is a relative value and must be normalised using data obtained from other techniques. It is interesting to compare these cross sections obtained at low collision energies with rate coefficients obtained at low temperatures by the CRESU technique. If there is a negligible rotational state dependence of the reaction cross section / rate coefficient, and, for multiple product channels, if the branching ratio for the monitored product in the case of the crossed beam experiments is collision energy independent, then the temperature dependence $n$ of the thermal rate coefficient $k$ (where $k \propto T^{n}$ ) and the collision energy dependence $\alpha$ of the reaction cross section $\sigma$ (where $\sigma \propto E_{T}^{\alpha}$ ) should be related by the simple formula $n=\alpha+1 / 2$. This has been found to be the case, within experimental error, for a number of reactions, including for example the reactions of $\mathrm{C}\left({ }^{3} \mathrm{P}\right)$ atoms with $\mathrm{O}_{2}$ and $\mathrm{NO}$ [103]. 


\section{PERSPECTIVES, FUTURE DIRECTIONS AND CONCLUSION}

\subsection{Charges species}

With respect to ion-molecule reactions, the development of pulsed supersonic flows in Rennes will offer the possibility of achieving very low pressures in the supersonic flow due to the high pumping speed available $(\sim 30000 \mathrm{~m} 3 / \mathrm{h})$ and therefore will open the way to a revival of the original ion-molecule CRESU apparatus by the use of a selective injection of ions which will be directly derived from expertise gained previously $[53,54]$. Studies involving expensive deuterated species could also be of great interest. Ion-atom reactions are an important family of processes for which low temperature exploration is in its infancy. There is however a significant amount of data that were obtained at room temperature [104] for $\mathrm{H}, \mathrm{N}$ and $\mathrm{O}$ atoms using the SIFT apparatus. The first attempt made by D. Gerlich, using an innovative ion trap, concerning the reactivity of $\mathrm{H}$ atoms at temperatures below $100 \mathrm{~K}$ is clearly promising [45, 49] and could be extended to N, O or possibly metal atoms. This kind of studies could also be considered in the CRESU apparatus in Rennes provided that a stable and dense atom source is implemented.

The very recent discovery of anions in several astrophysical environments has excited the astrochemical community and it is clear that there is now a real desire to include electron attachment reactions in the models based on reliable laboratory data. The field is completely virgin at the temperatures of dense interstellar clouds and present developments of the CRESU technique should allow tackling this interesting topic. One of the main goals should be the study of the electron attachment of PAHs using a cold source of electrons. By coupling laser and mass spectrometry techniques it should be also possible to study attachment to radicals such as those observed in space, including smaller ones such as $\mathrm{CN}$ which could act as precursors for bigger species. The study of anion-molecule reactions at very low temperature is henceforth another strong expectation.

Mastering the production of cold electrons in the CRESU supersonic flow could also open up the possibility of measuring thermal rate coefficients for the dissociative recombination of polyatomic ions at temperatures below $77 \mathrm{~K}$, which will be of considerable interest for interstellar chemistry. With respect to ion storage ring machines, the situation changed significantly quite recently. ASTRID is now functioning as a dedicated full time synchrotron radiation source whereas TARN II and CRYRING are no longer operational. The TSR in Heidelberg is still working but only for light molecular ions and it is not a user facility. Fortunately, a new facility is due to become operational at the Institute of Modern Physics in Lanzhou, China in 2012-2013. This user facility will be ideally suited to measurements of heavy hydrocarbon ions and to the more complete identification of branching channels. Electrostatic storage rings could be another alternative for the future as some attempts have been successful in studying the dissociative recombination of biologically interesting ions such as peptides [105] (KEK, Tsukuba, Japan). A lot of work has still to be done in this field, however, especially for large molecules including PAHs for which a series of measurements are presently available only at room temperature [106, 107].

\subsection{Neutrals}

Most of the presently available studies concerning neutral neutral reactions are related to small systems i.e. atomic or simple molecular radicals and reagents but there are still several routes that remain unexplored.

As a case in point, the chemistry of sulfur is completely unknown at very low temperatures, although molecules such as $\mathrm{SO}$ or $\mathrm{SO}_{2}$ are routinely used to trace shocks in the ISM. The reactivity of sulfur atoms is certainly of interest in the production of more complex sulfur-bearing molecules. A recent effort has been done in Rennes and Perugia (Italy) to investigate the reactivity of atomic sulphur in its metastable state ${ }^{1} \mathrm{D}$ with small hydrocarbons [108, 109].

A much more difficult task is the experimental study of radical-radical reactions. After the pioneering work led in Birmingham down to $100 \mathrm{~K}$ concerning reactions of $\mathrm{OH}$ with $\mathrm{O}$ and $\mathrm{N}$ atoms [110] in 
a cryogenic cell, two successful attempts have been done at lower temperatures using the CRESU technique quite recently. The $\mathrm{O}+\mathrm{OH}$ reaction had been investigated in the temperature range 39$300 \mathrm{~K}$ in Birmingham [111] whereas the mini CRESU available in Bordeaux was used for the study of $\mathrm{N}+\mathrm{NO}$ in the temperature range 48-211 K [112]. The main difficulty of this kind of experiment lies in generating a large and quantifiable concentration of atoms in the flow in addition to producing the molecular radical. Future work may focus on reactions such as $\mathrm{N}+\mathrm{OH}, \mathrm{O}+\mathrm{CN}, \mathrm{O}+\mathrm{CH}$ which are all of particular interest in interstellar chemistry.

Another important aspect revealed by modern observational astronomy is the complexity of the existing interstellar molecules. Understanding how this complexity may be generated and to what level it can be brought is clearly a central issue. For example the reactivity of PAHs with other molecules is of particular interest because insertion mechanisms could contribute to the building up of new species containing an increasing number of aromatic cycles. The study of dimerisation and eventually nucleation of PAHs can also give new insights in the generation of nanoparticles and by extension interstellar dust. On another hand, several precursors of prebiotic molecules have been detected in space: the first interstellar sugar glycolaldehyde $\left(\mathrm{CH}_{2} \mathrm{OHCHO}\right)$; ethylene glycol $\left(\mathrm{HOCH}_{2} \mathrm{CH}_{2} \mathrm{OH}\right)$, a hydrogenated sugar better known as antifreeze; acetamide, $\mathrm{CH}_{3} \mathrm{CONH}_{2}$, the largest interstellar molecule with a peptide bond, and amino acetonitrile $\left(\mathrm{NH}_{2} \mathrm{CH}_{2} \mathrm{CN}\right)$, a direct precursor of the simplest amino acid glycine. Present experimental work has shown that reactions with carbon containing radicals usually lead to products that are carbon enriched with respect to the reagent [18] and then these processes can be important routes to the construction of ring species many of which are of biological importance. For instance, a very recent work concerning the reaction of the cyanide radical $\mathrm{CN}$ with the 1,3butadiene molecule, $\mathrm{H}_{2} \mathrm{CCHCHCH}$, provided some evidence that the aromatic pyridine $\mathrm{C}_{5} \mathrm{H}_{5} \mathrm{~N}$ could be produced although in small quantity [113]. Therefore reactions of simple stable $\mathrm{C}_{2}, \mathrm{C}_{3}$ and $\mathrm{C}_{4}$-based hydrocarbons with radicals should also be completed. It must also be said here that routes involving charged species are to be explored as well for the production of these molecules.

Identification of the products and the quantification of the branching ratios are vital for a complete understanding of a reactive process. Mass spectrometry is here a powerful tool that allowed tackling this problem for ion-molecule studies. For neutral neutral reactions this aspect is much more difficult to explore using the available experimental techniques. Although products can be identified by several methods such as LIF or mass spectrometry the determination of their concentration is not usually possible. Nevertheless, reactions for which only one exit channel is open can be used as references to determine the product branching ratio of another process. This idea has been used in the groups of Seakins [114-118] and Loison [119-125] to obtain the branching ratios of channels producing hydrogen atoms. Calibration reactions such as $\mathrm{CN}+\mathrm{H}_{2} \rightarrow \mathrm{HCN}+\mathrm{H}, \mathrm{CH}+\mathrm{CH}_{4} \rightarrow \mathrm{C}_{2} \mathrm{H}_{4}+\mathrm{H}$ or $\mathrm{C}+\mathrm{C}_{2} \mathrm{H}_{4} \rightarrow \mathrm{C}_{3} \mathrm{H}_{3}+\mathrm{H}$ can be used where $\mathrm{H}$ atoms are detected by VUV LIF. These studies however are only available at room temperature and also at $195 \mathrm{~K}$ for some cases. It would be worthwhile to extend them to interstellar temperatures obviously.

The development of infrared lasers opens new possibilities for the determination of branching ratios. Multipass cells can be coupled to infrared light to detect molecular radicals by absorption techniques and quantify their concentration. Using this method, Hershberger and his co-workers obtained a significant amount of results at room temperature and above [126]. One of the major problems here lies in the vibrational population of the product molecules which may not be in equilibrium, thus perturbing the infrared absorption measurements. This has been solved by using $\mathrm{SF}_{6}$ as a relaxant, a technique which should not be applicable to CRESU supersonic flows because of potential polymerisation of large concentrations of $\mathrm{SF}_{6}$ during the expansion.

A more promising technique lies in the photoionisation of products at threshold using VUV light and collection of the ions by mass spectrometry techniques. VUV can be generated by non-linear mixing laser techniques, a method which is rather limited, however, because of the restricted tunability of the generated light. Furthermore, the photon flux is generally rather small. A more appropriate tool for this kind of study is light produced from a synchrotron. Recent studies have been undertaken at 
room temperature coupling a slow flow cell reactor to the ALS synchrotron in Berkeley [127-132]. By scanning the VUV light it is possible to detect products according to their ionisation potential which allows one to identify isomers. Provided that ionisation cross sections are known, the relative concentration of the products and therefore the branching ratios can be extracted from the experiment. The successful development of a pulsed version of the CRESU apparatus in Rennes allowing the study of reaction kinetics down to $c a$. $20 \mathrm{~K}$ opens now the door to the possibility of building a reduced transportable version of the CRESU apparatus which could be coupled to a synchrotron (e.g. SOLEIL in France) and then perform isomer sensitive product detection at low temperature.

Beyond the study of products, the pulsed CRESU available in Rennes will allow investigating reactions involving expensive reagents such as deuterated species because of the significant reduction of gas consumption (presently a factor a 8 which is expected to increase to 20 in the future). For the same reason, it will be also possible to consider reactions for which the reagent must be synthesized and therefore only a limited quantity will generally be available. Potential examples are $\mathrm{HC}_{3} \mathrm{~N}$ and $\mathrm{C}_{4} \mathrm{H}_{2}$.

\section{CONCLUSION}

As we hope to have demonstrated in the preceding pages, there has been a considerable work that has been carried out for the last twenty years. The field of gas kinetics at low temperature is very active and still possesses many unexplored areas however. The development of new technologies will, no doubt, enable experimentalists to push the limits even further, and exciting results are to be expected in the near future.

\section{References}

[1] P.F. Goldsmith and W.D. Langer, "Molecular Cooling and Thermal Balance of Dense Interstellar Clouds" Astrophysical Journal, 222, 881-895 (1978).

[2] J. Rawlings, "The role of astrochemistry in low mass star formation" Astrophys. \& Space Sc., 285[3-4], 777-789 (2003).

[3] D.E. Woon, "The Astrochymist” www. astrochymist. org/astrochymist_ism. html, (2010).

[4] S. Iglesias Groth, A. Manchado, D.A. García-Hernández, J.I. González-Hernández, and D.L. Lambert, "Evidence for the Naphthalene Cation in A Region of the Interstellar Medium with Anomalous Microwave Emission" Astrophys. J. Lett., 685[1], L55-L58 (2008).

[5] S. Iglesias-Groth, A. Manchado, R. Rebolo, J.I. González-Hernández, D.A. García-Hernández, and D.L. Lambert, "A search for interstellar anthracene towards the Perseus anomalous microwave emission region” Mon. Not. R. Astron. Soc., 409[2], 880 (2010).

[6] I.W.M. Smith, E. Herbst, and Q. Chang, "Rapid neutral-neutral reactions at low temperatures: a new network and first results for TMC-1" Mon. Not. R. Astron. Soc., 350[1], 323-330 (2004).

[7] J. Woodall, M. Agúndez, A.J. Markwick-Kemper, and T.J. Millar, "The UMIST database for astrochemistry 2006" Astron. \& Astrophys., 466[3], 1197-1204 (2007).

[8] J.I. Moses, B. Bezard, E. Lellouch, G.R. Gladstone, H. Feuchtgruber, and M. Allen, "Photochemistry of Saturn's atmosphere - I. Hydrocarbon chemistry and comparisons with ISO observations" Icarus, 143[2], 244-298 (2000).

[9] E.H. Wilson and S.K. Atreya, "Current state of modeling the photochemistry of Titan's mutually dependent atmosphere and ionosphere" J. Geophys. Res, 109, E06002 (2004).

[10] J.I. Moses and T.K. Greathouse, "Latitudinal and seasonal models of stratospheric photochemistry on Saturn: Comparison with infrared data from IRTF/TEXES” J. Geophys. Res. E, 110[E9], E09007 (2005).

[11] S. Lebonnois, "Benzene and aerosol production in Titan and Jupiter's atmospheres: a sensitivity study" Planet. Space Sc., 53[5], 486-497 (2005). 
[12] V.A. Krasnopolsky, "Photochemistry of the martian atmosphere: Seasonal, latitudinal, and diurnal variations" Icarus, 185[1], 153-170 (2006).

[13] V.A. Krasnopolsky, “A photochemical model of Titan's atmosphere and ionosphere" Icarus, 201[1], 226-256 (2009).

[14] V. Vuitton, R.V. Yelle, and P. Lavvas, "Composition and chemistry of Titan's thermosphere and ionosphere" Phil. Trans. Roy. Soc. A, 367[1889], 729-741 (2009).

[15] J. Woodall, M. Agundez, A.J. Markwick-Kemper, and T.J. Millar, "The UMIST database for astrochemistry 2006" Astronomy And Astrophysics, 466[3], 1197-1204 (2007).

[16] E. Herbst and V. Wakelam, "The OSU Chemical Network" http://www. physics. ohio-state. edu/ eric/research. html, (2008).

[17] J. Woodall, M. Agúndez, A.J. Markwick-Kemper, and T.J. Millar, "The UMIST Database for Astrochemistry" http://www. udfa. net/, (2008).

[18] A. Canosa, F. Goulay, I.R. Sims, and B.R. Rowe, "Gas Phase Reactive Collisions at very Low Temperature: Recent Experimental Advances and Perspectives" in Low Temperatures and Cold Molecules, p 55-120, (Ed. Imperial College Press, 2008).

[19] B.R. Rowe and J.B. Marquette, "CRESU Study of ion molecule reactions" Int. J. Mass Spectrom. Ion Processes, 80, 239-254 (1987).

[20] M.A. Smith, "Ion Molecule Reaction Dynamics at very low Temperatures" in Unimolecular and Bimolecular Reaction Dynamics, p 183-251, (Ed. C. Y. Ng, T. Baer, and I. Powis, John Wiley \& Sons Ltd, New York, 1994).

[21] A. Chutjian, A. Garscadden, and J.M. Wadehra, "Electron attachment to molecules at low electron energies" Phys. Rep., 264[6], 393-470 (1996).

[22] NIST, "NIST Chemical Kinetics Database on the Web: Standard Reference Database 17, Version 7.0 (Web Version), Release 1.5” http://kinetics. nist. gov/kinetics/index. jsp, (2000).

[23] V.G. Anicich, “An Index of the Literature for Bimolecular Gas Phase Cation-Molecule Reaction Kinetics" JPL Publication, 19, 1-1194 (2003).

[24] A.I. Florescu-Mitchell and J.B.A. Mitchell, "Dissociative recombination" Phys. Rep., 430[5-6], 277-374 (2006).

[25] I.W.M. Smith, "Laboratory studies of atmospheric reactions at low temperatures" Chem. Rev., 103[12], 4549-4564 (2003).

[26] M.J. Frost, P. Sharkey, and I.W.M. Smith, "Reaction Between OH (OD) Radicals and CO at Temperatures down to $80 \mathrm{~K}$ - Experiment and Theory” J. Phys. Chem., 97[47], 12254-12259 (1993).

[27] K. Giles, N.G. Adams, and D. Smith, "A Study of the Reactions of $\mathrm{H}_{3}^{+}, \mathrm{H}_{2} \mathrm{D}^{+}, \mathrm{HD}_{2}^{+}$, and $\mathrm{D}_{3}^{+}$ with $\mathrm{H}_{2}$, $\mathrm{HD}$ and $\mathrm{D}_{2}$ Using A Variable-Temperature Selected Ion Flow Tube" J. Phys. Chem., 96[19], 7645-7650 (1992).

[28] L.K. Randeniya and M.A. Smith, "A Study of Molecular Supersonic Flow using the Generalized Boltzmann Equation” J. Chem. Phys., 93[1], 661-673 (1990).

[29] G. Scoles, Atomic and molecular beam methods, (Oxford University Press, 1988).

[30] C. Naulin and M. Costes, "Crossed Beam Study of the $A l\left({ }^{2} P_{1 / 2,3 / 2}\right)+O_{2}\left(X^{3} \Sigma_{g}^{-}\right) \rightarrow$ AlO $\left(X^{2} \Sigma^{+}\right)+O\left({ }^{3} P_{J}\right)$ " Chem. Phys. Lett., 310, 231-239 (1999).

[31] N. Balucani, G. Capozza, F. Leonori, E. Segoloni, and P. Casavecchia, "Crossed molecular beam reactive scattering: from simple triatomic to multichannel polyatomic reactions" IRPC, 25[1-2], 109-163 (2006).

[32] R.A. Phaneuf, C.C. Havener, G.H. Dunn, and A. Muller, "Merged-beams experiments in atomic and molecular physics" Reports on Progress in Physics, 62[7], 1143-1180 (1999).

[33] D. Gerlich, "Experimental Investigations of Ion-Molecule Reactions Relevant to Interstellar Chemistry" J. Chem. Soc. Faraday Trans., 89[13], 2199-2208 (1993).

[34] M. Larsson, "Dissociative recombination with ion storage rings" Annu. Rev. Phys. Chem., 48, 151-179 (1997). 
[35] G. Dupeyrat, J.B. Marquette, and B.R. Rowe, "Design and testing of axisymmetric nozzles for ion molecule reaction studies between $20 \mathrm{~K}$ and $160 \mathrm{k}$ " Phys. Fl., 28[5], 1273-1279 (1985).

[36] I.R. Sims, J.L. Queffelec, A. Defrance, C. Rebrion-Rowe, D. Travers, P. Bocherel, B.R. Rowe, and I.W.M. Smith, "Ultra-low temperature kinetics of neutral-neutral reactions : The technique, and results for the reactions $\mathrm{CN}+\mathrm{O}_{2}$ down to $13 \mathrm{~K}$ and $\mathrm{CN}+\mathrm{NH}_{3}$ down to $25 \mathrm{~K}$." J. Chem. Phys., 100[6], 4229-4241 (1994).

[37] P.L. James, I.R. Sims, and I.W.M. Smith, "Total and state-to-state rate coefficients for rotational energy transfer in collisions between $N O\left(X^{2} \Pi\right)$ and He at temperatures down to $15 \mathrm{~K}^{\prime}$ " Chem. Phys. Lett., 272[5-6], 412-418 (1997).

[38] N. Daugey, P. Caubet, B. Retail, M. Costes, A. Bergeat, and G. Dorthe, "Kinetic measurements on methylidyne radical reactions with several hydrocarbons at low temperatures" Phys. Chem. Chem. Phys., 7[15], 2921-2927 (2005).

[39] D.B. Atkinson and M.A. Smith, "Design and Characterization of Pulsed Uniform Supersonic Expansions for Chemical Applications" Rev. Sci. Instrum., 66[9], 4434-4446 (1995).

[40] S. Lee, R.J. Hoobler, and S.R. Leone, "A Pulsed Laval Nozzle Apparatus with Laser Ionization Mass Spectrometry for Direct Measurements of Rate Coefficients at low Temperatures with Condensable Gases" Rev. Sci. Instrum., 71[4], 1816-1823 (2000).

[41] T. Spangenberg, S. Kohler, B. Hansmann, U. Wachsmuth, B. Abel, and M.A. Smith, "Lowtemperature reactions of $\mathrm{OH}$ radicals with propene and isoprene in pulsed laval nozzle expansions" J. Phys. Chem. A, 108[37], 7527-7534 (2004).

[42] S.E. Taylor, A. Goddard, M.A. Blitz, P.A. Cleary, and D.E. Heard, "Pulsed Laval nozzle study of the kinetics of $\mathrm{OH}$ with unsaturated hydrocarbons at very low temperatures" Phys. Chem. Chem. Phys., 10[3], 422-437 (2008).

[43] S. Morales, PhD: "Le Hacheur Aérodynamique: un nouvel instrument dédié aux processus réactionnels à ultra-basse température", December 14th 2009, Université de Rennes 1 (France)

[44] S. Petrie and D.K. Bohme, "Mass spectrometric approaches to interstellar chemistry" Mod. Mass Spectrom., 225, 37-75 (2003).

[45] D. Gerlich, "The Study of Cold Collisions using Ion Guides and Traps" in Low Temperatures and Cold Molecules, p 121-174, (Ed. Imperial College Press, 2008).

[46] N.G. Adams and D. Smith, "The Selected Ion Flow Tube: a Technique for studying Ion-Neutral reactions" Int. J. Mass Spectrom. Ion Phys., 21, 349-359 (1976).

[47] H. Bohringer and F. Arnold, "Studies of Ion Molecule Reactions, Ion Mobilities, and Their Temperature-Dependence to Very Low-Temperatures Using A Liquid-Helium-Cooled Ion Drift Tube" Int. J. Mass Spectrom. Ion Processes, 49[1], 61-83 (1983).

[48] S.E. Barlow, J.A. Luine, and G.H. Dunn, "Measurement of Ion Molecule Reactions between 10 and 20 K” Int. J. Mass Spectrom. Ion Processes, 74, 97-128 (1986).

[49] D. Gerlich and M. Smith, "Laboratory astrochemistry: studying molecules under inter- and circumstellar conditions" Phys. Scripta, 73[1], C25-C31 (2006).

[50] G. Borodi, A. Luca, and D. Gerlich, "Reactions of $\mathrm{CO}_{2}^{+}$with $\mathrm{H}, \mathrm{H}_{2}$ and deuterated analogues" Int. J. Mass Spectrom., 280[1-3], 218-225 (2009).

[51] S.E. Barlow, G.H. Dunn, and M. Schauer, "Radiative Association of $\mathrm{CH}_{3}^{+}$and $\mathrm{H}_{2}$ at $13 \mathrm{~K}$ " Phys. Rev. Lett., 52[11], 902-905 (1984).

[52] D. Gerlich and S. Horning, "Experimental Investigation of Radiative Association Processes as related to Interstellar Chemistry" Chem. Rev., 92, 1509-1539 (1992).

[53] B.R. Rowe, J.B. Marquette, and C. Rebrion, "Mass selected ion-molecule reactions at very low temperatures: the CRESUS apparatus" J. Chem. Soc. Faraday Trans. II, 85[10], 1631-1641 (1989).

[54] T. Speck, T. Mostefaoui, D. Travers, and B.R. Rowe, "Pulsed Injection of Ions into the CRESU Experiment" Int. J. Mass Spectrom., 208[1-3], 73-80 (2001). 
[55] M. Hawley, T.L. Mazely, L.K. Randeniya, R.S. Smith, X.K. Zeng, and M.A. Smith, “A Free Jet Flow Reactor for Ion Molecule Reaction Studies at Very Low Energies" Int. J. Mass Spectrom. Ion Processes, 97[1], 55-86 (1990).

[56] M.A. Smith and M. Hawley, "Ion Chemistry at Extremely low Temperatures: a Free Jet Expansion Approach" Adv. Gas Phase Ion. Chem., 1, 167-202 (1992).

[57] A. Kalamarides, R.W. Marawar, M.A. Durham, B.G. Lindsay, K.A. Smith, and F.B. Dunning, "Use of Rydberg Atoms to Probe Negative-Ion Lifetimes" J. Chem. Phys., 93[6], 4043-4046 (1990).

[58] A. Kalamarides, R.W. Marawar, X. Ling, C.W. Walter, B.G. Lindsay, K.A. Smith, and F.B. Dunning, "Negative-Ion Production in Collisions Between $\mathrm{K}(\mathrm{Nd})$ Rydberg Atoms and $\mathrm{CF}_{3} \mathrm{Br}$ and $\mathrm{CF}_{2} \mathrm{Br}_{2}$ " J. Chem. Phys., 92[3], 1672-1676 (1990).

[59] E. Alge, N.G. Adams, and D. Smith, "Rate Coefficients for the Attachment Reactions of Electrons with $\mathrm{c}-\mathrm{C}_{7} \mathrm{~F}_{14}, \mathrm{CH}_{3} \mathrm{Br}, \mathrm{CF}_{3} \mathrm{Br}, \mathrm{CH}_{2} \mathrm{Br}_{2}$ and $\mathrm{CH}_{3} \mathrm{I}$ Determined Between $200 \mathrm{~K}$ and $600 \mathrm{~K}$ Using the Falp Technique” J. Phys. B, 17[18], 3827-3833 (1984).

[60] D. Smith, N.G. Adams, and E. Alge, "Attachment Coefficients for the Reactions of Electrons with $\mathrm{CCl}_{4}, \mathrm{CCl}_{3} \mathrm{~F}, \mathrm{CCl}_{2} \mathrm{~F}_{2}, \mathrm{CHCl}_{3}, \mathrm{Cl}_{2}$ and $\mathrm{SF}_{6}$ Determined Between $200 \mathrm{~K}$ and $600 \mathrm{~K}$ Using the Falp Technique" J. Phys. B, 17[3], 461-472 (1984).

[61] J.L. Le Garrec, O. Sidko, J.L. Queffelec, S. Hamon, J.B.A. Mitchell, and B.R. Rowe, "Experimental studies of cold electron attachment to $\mathrm{SF}_{6}, \mathrm{CF}_{3} \mathrm{Br}$ and $\mathrm{CCl}_{2} \mathrm{~F}_{2}$ " J. Chem. Phys., 107[1], 54-63 (1997).

[62] F. Goulay, C. Rebrion-Rowe, S. Carles, J.L. Le Garrec, and B.R. Rowe, "Electron attachment on HI and DI in a uniform supersonic flow: Thermalization of the electrons" J. Chem. Phys., 121[3], 1303-1308 (2004).

[63] J.E. Wahlund, R. Bostrom, G. Gustafsson, D.A. Gurnett, W.S. Kurth, A. Pedersen, T.F. Averkamp, G.B. Hospodarsky, A.M. Persoon, P. Canu, F.M. Neubauer, M.K. Dougherty, A.I. Eriksson, M.W. Morooka, R. Gill, M. Andre, L. Eliasson, and W. Muller, I, "Cassini measurements of cold plasma in the ionosphere of Titan" Science, 308[5724], 986-989 (2005).

[64] N.G. Adams, V. Poterya, and L.M. Babcock, "Electron molecular ion recombination: Product excitation and fragmentation" Mass Spectrom. Rev., 25[5], 798-828 (2006).

[65] E. Alge, N.G. Adams, and D. Smith, "Measurements of the Dissociative Recombination Coefficients of $\mathrm{O}_{2}^{+}, \mathrm{NO}^{+}$and $\mathrm{NH}_{4}^{+}$in the Temperature-Range 200-600 K" J. Phys. B, 16[8], 1433-1444 (1983).

[66] N.G. Adams, D. Smith, and E. Alge, "Measurements of Dissociative Recombination Coefficients of $\mathrm{H}_{3}^{+}, \mathrm{HCO}^{+}, \mathrm{N}_{2} \mathrm{H}^{+}$, and $\mathrm{CH}_{5}^{+}$at $95 \mathrm{~K}$ and $300 \mathrm{~K}$ Using the Falp Apparatus" J. Chem. Phys., 81[4], 1778-1784 (1984).

[67] J.L. Mclain, V. Poterya, C.D. Molek, L.M. Babcock, and N.G. Adams, "Flowing afterglow studies of the temperature dependencies for dissociative recombination of $\mathrm{O}_{2}^{+}, \mathrm{CH}_{5}^{+}, \mathrm{C}_{2} \mathrm{H}_{5}^{+}$, and $\mathrm{C}_{6} \mathrm{H}_{7}^{+}$with electrons" J. Phys. Chem. A, 108[32], 6704-6708 (2004).

[68] N.G. Adams, C.R. Herd, and D. Smith, "Development of the Flowing Afterglow Langmuir Probe Technique for Studying the Neutral Products of Dissociative Recombination Using Spectroscopic Techniques - OH Production in the $\mathrm{HCO}^{+}+$e Reaction” J. Chem. Phys., 91[2], 963-973 (1989).

[69] N.G. Adams, C.R. Herd, M. Geoghegan, D. Smith, A. Canosa, J.C. Gomet, B.R. Rowe, J.L. Queffelec, and M. Morlais, "Laser induced fluorescence and vacuum ultraviolet spectroscopic studies of $H$ atom production in the dissociative recombination of some protonated ions" J. Chem. Phys., 94[7], 4852-4857 (1991).

[70] R.E. Rosati, R. Johnsen, and M.F. Golde, "Absolute yields of $C O\left(a^{\prime}{ }^{3} \Sigma^{+}, d^{3} \Delta_{i}, e^{3} \Sigma^{-}\right)+O$ from the dissociative recombination of $\mathrm{CO}_{2}^{+}$ions with electrons" J. Chem. Phys., 119[22], 11630-11635 (2003). 
[71] R.E. Rosati, M.P. Skrzypkowski, R. Johnsen, and M.F. Golde, "Yield of excited CO molecules from dissociative recombination of $\mathrm{HCO}^{+}$and $\mathrm{HOC}^{+}$ions with electrons" J. Chem. Phys., 126[15], 154302 (2007).

[72] R.E. Rosati, R. Johnsen, and M.F. Golde, "Yield of electronically excited $N_{2}$ molecules from the dissociative recombination of $\mathrm{N}_{2} \mathrm{H}^{+}$with e" J. Chem. Phys., 120[17], 8025-8030 (2004).

[73] T. Gougousi, R. Johnsen, and M.F. Golde, "Yield determination of $O H(v=0,1)$ radicals produced by the electron-ion recombination of $\mathrm{H}_{3} \mathrm{O}^{+}$ions" J. Chem. Phys., 107[7], 2430-2439 (1997).

[74] T. Gougousi, R. Johnsen, and M.F. Golde, "Yield determination of $O H(v=0,1)$ radicals produced by the electron-ion recombination of protonated molecules" J. Chem. Phys., 107[7], 2440-2443 (1997).

[75] R.E. Rosati, D. Pappas, R. Johnsen, and M.F. Golde, "Yield of electronically excited CN molecules from the dissociative recombination of $\mathrm{HNC}^{+}$with electrons - art. no. 154303" J. Chem. Phys., 126[15], 154303 (2007).

[76] C.D. Molek, J.L. Mclain, V. Poterya, and N.G. Adams, "A remeasurement of the products for electron recombination of $\mathrm{N}_{2} \mathrm{H}^{+}$using a new technique: No significant NH plus N production" J. Phys. Chem. A, 111[29], 6760-6765 (2007).

[77] C.D. Molek, V. Poterya, N.G. Adams, and J.L. Mclain, "Development of a novel technique for quantitatively determining the products of electron-ion dissociative recombination" Int. J. Mass Spectrom., 285[1-2], 1-11 (2009).

[78] M. Larsson and A.E. Orel, Dissociative Recombination of Molecular Ions, (Cambridge University Press, 2008).

[79] A. Auerbach, R. Cacak, R. Caudano, T.D. Gaily, C.J. Keyser, J.Wm. McGowan, J.B.A. Mitchell, and S.F.J. Wilk, "Merged Electron-Ion Beam Experiments. I. Method and Measurements of $\left(e-H_{2}^{+}\right)$and $\left(e-H_{3}^{+}\right)$Dissociative Recombination Cross Sections" J. Phys. B, 10[18], 3797-3820 (1977).

[80] J.B.A. Mitchell, J.L. Forand, C.T. Ng, D.P. Levac, R.E. Mitchell, P.M. Mul, W. Claeys, A. Sen, and J.Wm. McGowan, "Measurement of the Branching Ratio for the Dissociative Recombination of $\mathrm{H}_{3}^{+}+e$ " Phys. Rev. Lett., 51[10], 885-888 (1983).

[81] S. Datz, G. Sundstrom, C. Biedermann, L. Brostrom, H. Danared, S. Mannervik, J.R. Mowat, and M. Larsson, "Branching-Processes in the Dissociative Recombination of $\mathrm{H}_{3}^{+}$" Phys. Rev. Lett., 74[6], 896-899 (1995).

[82] A. Neau, A. AlKhalili, S. Rosen, A. LePadellec, A.M. Derkatch, W. Shi, L. Vikor, M. Larsson, J. Semaniak, R. Thomas, M.B. Nagard, K. Andersson, H. Danared, and M. afUgglas, "Dissociative recombination of $\mathrm{D}_{3} \mathrm{O}^{+}$and $\mathrm{H}_{3} \mathrm{O}^{+}$: Absolute cross sections and branching ratios" J. Chem. Phys., 113[5], 1762-1770 (2000).

[83] J.B.A. Mitchell, C. Rebrion-Rowe, J.L. Le Garrec, G. Angelova, H. Bluhme, K. Seiersen, and L.H. Andersen, "Branching Ratios for the Dissociative Recombination of Hydrocarbon Ions. I: The case of $\mathrm{C}_{4} \mathrm{H}_{9}^{+}$and $\mathrm{C}_{4} \mathrm{H}_{5}^{+}$” Int. J. Mass Spectrom., 227[2], 273-279 (2003).

[84] G. Angelova, O. Novotny, J.B.A. Mitchell, C. Rebrion-Rowe, J.L. Le Garrec, H. Bluhme, A. Svendsen, and L.H. Andersen, "Branching Ratios for the Dissociative Recombination of Hydrocarbon Ions. III: The case of $C_{3} H_{n}^{+}(n=1-8)$ ” Int. J. Mass Spectrom., 235[1], 7-13 (2004).

[85] G. Angelova, O. Novotny, J.B.A. Mitchell, C. Rebrion-Rowe, J.L. Le Garrec, H. Bluhme, K. Seiersen, and L.H. Andersen, "Branching Ratios for the Dissociative Recombination of Hydrocarbon Ions.II The Cases of $\mathrm{C}_{4} \mathrm{H}_{n}^{+}(n=1-9)$.” Int. J. Mass Spectrom., 232[2], 195-203 (2004).

[86] M. Larsson, A. Ehlerding, W.D. Geppert, F. Hellberg, S. Kalhori, R.D. Thomas, N. Djuric, F. Osterdahl, G. Angelova, J. Semaniak, O. Novotny, S.T. Arnold, and A.A. Viggiano, "Rate constants and branching ratios for the dissociative recombination of $C_{3} D_{7}^{+}$and $C_{4} D_{9}^{+}$", J. Chem. Phys., 122[15], 6101 (2005). 
[87] E. Herbst and W. Klemperer, "The Formation and Depletion of Molecules in Dense Interstellar Clouds" Astrophysical Journal, 185, 505-533 (1973).

[88] F. Goulay, C. Rebrion-Rowe, J.L. Le Garrec, S.D. Le Picard, A. Canosa, and B.R. Rowe, “The Reaction of Anthracene with $\mathrm{OH}$ Radicals: an Experimental Study of the kinetics between $58 \mathrm{~K}$ and 470 K” J. Chem. Phys., 122[10], 104308 (2005).

[89] F. Goulay, C. Rebrion-Rowe, L. Biennier, S.D. Le Picard, A. Canosa, and B.R. Rowe, "The Reaction of Anthracene with CH Radicals: an Experimental Study of the Kinetics between $58 \mathrm{~K}$ and 470 K” J. Phys. Chem. A, 110[9], 3132-3137 (2006).

[90] I.R. Sims, J.L. Queffelec, A. Defrance, C. Rebrion-Rowe, D. Travers, B.R. Rowe, and I.W.M. Smith, "Ultralow temperature kinetics of neutral-neutral reactions : the reaction $\mathrm{CN}+\mathrm{O}_{2}$ down to 26 K" J. Chem. Phys., 97[11], 8798-8800 (1992).

[91] C. Mullen and M.A. Smith, "Low temperature $N H\left(X^{3} \Sigma\right)$ radical reactions with $N O$, saturated, and unsaturated hydrocarbons studied in a pulsed supersonic laval nozzle flow reactor between 53 and 188 K" J. Phys. Chem. A, 109[7], 1391-1399 (2005).

[92] C. Mullen and M.A. Smith, "Temperature dependence and kinetic isotope effects for the OH $+\mathrm{HBr}$ reaction and $\mathrm{H} / \mathrm{D}$ isotopic variants at low temperatures (53-135 K) measured using a pulsed supersonic laval nozzle flow reactor" J. Phys. Chem. A, 109[17], 3893-3902 (2005).

[93] I.W.M. Smith, A.M. Sage, N.M. Donahue, E. Herbst, and D. Quan, "The temperaturedependence of rapid low temperature reactions: experiment, understanding and prediction" Faraday Discuss., 133, 137-156 (2006).

[94] I.R. Sims, J.L. Queffelec, D. Travers, B.R. Rowe, L.B. Herbert, J. Karthäuser, and I.W.M. Smith, "Rate constants for the Reactions of CN with Hydrocarbons at Low and Ultra-low Temperatures " Chem. Phys. Lett., 211[4-5], 461-468 (1993).

[95] E.E. Greenwald, S.W. North, Y. Georgievskii, and S.J. Klippenstein, "A two transition state model for radical-molecule reactions: A case study of the addition of $\mathrm{OH}$ to $\mathrm{C}_{2} \mathrm{H}_{4}$ " J. Phys. Chem. A, 109[27], 6031-6044 (2005).

[96] Y. Georgievskii and S.J. Klippenstein, "Strange kinetics of the $C_{2} H_{6}+C N$ reaction explained" J. Phys. Chem. A, 111[19], 3802-3811 (2007).

[97] H. Sabbah, L. Biennier, I.R. Sims, Y. Georgievskii, S.J. Klippenstein, and I.W.M. Smith, "Understanding reactivity at very low temperatures: The reactions of oxygen atoms with alkenes" Science, 317[5834], 102-105 (2007).

[98] N.M. Donahue, "Reaction barriers: Origin and evolution" Chem. Rev., 103[12], 4593-4604 (2003).

[99] C. Berteloite, S.D. Le Picard, P. Birza, M.C. Gazeau, A. Canosa, Y. Benilan, and I.R. Sims, "Low temperature $(39 \mathrm{~K}-298 \mathrm{~K})$ kinetics study of the reactions of $\mathrm{C}_{4} \mathrm{H}$ radical with various hydrocarbons observed in Titan's atmosphere" Icarus, 194[2], 746-757 (2008).

[100] C. Berteloite, S.D. Le Picard, N. Balucani, A. Canosa, and I.R. Sims, “ Low Temperature Rate Coefficients for Reactions of the Butadiynyl Radical. $C_{4} H$, with Various Hydrocarbons. Part I: Reactions with Alkanes $\left(\mathrm{CH}_{4}, \mathrm{C}_{2} \mathrm{H}_{6}, \mathrm{C}_{3} \mathrm{H}_{8}, \mathrm{C}_{4} \mathrm{H}_{10}\right)$ " Phys. Chem. Chem. Phys., 12[15], 36663676 (2010).

[101] C. Berteloite, S.D. Le Picard, N. Balucani, A. Canosa, and I.R. Sims, "Low Temperature Rate Coefficients for Reactions of the Butadiynyl Radical, $C_{4} H$, with Various Hydrocarbons. Part II: Reactions with Alkenes (ethylene, propene, 1-butene), Dienes (allene, 1,3-butadiene) and Alkynes (acetylene, propyne and 1-butyne)" Phys. Chem. Chem. Phys., 12[15], 3677-3689 (2010).

[102] C. Berteloite, M. Lara, A. Bergeat, S.D. Le Picard, F. Dayou, K.M. Hickson, A. Canosa, C. Naulin, J.M. Launay, I.R. Sims, and M. Costes, "Kinetics and dynamics of the $S\left({ }^{1} D_{2}\right)+H_{2}$ reaction at very low temperatures and collision energies" Phys. Rev. Lett., 105(20) 203201 (2010). 
[103] W.D. Geppert, D. Reignier, T. Stoecklin, C. Naulin, M. Costes, D. Chastaing, S.D. LePicard, I.R. Sims, and I.W.M. Smith, "Comparison of the cross-sections and thermal rate constants for the reactions of $C\left({ }^{3} P_{J}\right)$ atoms with $\mathrm{O}_{2}$ and NO" Phys. Chem. Chem. Phys., 2[13], 2873-2881 (2000).

[104] T.P. Snow and V.M. Bierbaum, "Ion Chemistry in the Interstellar Medium" Ann. Rev. Anal. Chem., 1, 229-259 (2008).

[105] T. Tanabe, K. Noda, M. Saito, S. Lee, Y. Ito, and H. Takagi, "Resonant neutral-particle emission in collisions of electrons with peptide ions in a storage ring" Phys. Rev. Lett., 90[19] (2003).

[106] O. Novotny, B. Sivaraman, C. Rebrion-Rowe, D. Travers, L. Biennier, J.B.A. Mitchell, and B.R. Rowe, "Recombination of polycyclic aromatic hydrocarbon photoions with electrons in a flowing afterglow plasma" J. Chem. Phys., 123[10], 104303 (2005).

[107] L. Biennier, M.A. AlSayed-Ali, A. Foutel-Richard, O. Novotny, S. Carles, C. Rebrion-Rowe, and B.R. Rowe, "Laboratory measurements of the recombination of PAH ions with electrons: implications for the PAH charge state in interstellar clouds" Faraday Discuss., 133, 289-301 (2006).

[108] F. Leonori, R. Petrucci, N. Balucani, P. Casavecchia, M. Rosi, C. Berteloite, S.D. Le Picard, A. Canosa, and I.R. Sims, "Observation of organosulfur products (thiovinoxy, thioketene and thioformyl) in crossed-beam experiments and low temperature rate coefficients for the reaction $S\left({ }^{1} D\right)+C_{2} H_{4}$ " Phys. Chem. Chem. Phys., 11[23], 4701-4706 (2009).

[109] F. Leonori, R. Petrucci, N. Balucani, P. Casavecchia, M. Rosi, D. Skouteris, C. Berteloite, S.D. Le Picard, A. Canosa, and I.R. Sims, "Crossed-beam Dynamics, Low Temperature Kinetics and Theoretical Studies of the Reaction $S\left({ }^{1} D\right)+C_{2} H_{4}$ " J. Phys. Chem. A, 113[52], 15328-15345 (2009).

[110] I.W.M. Smith and Stewart D.W.A., "Low-temperature kinetics of reactions between neutral free radicals. Rate constants for the reactions of $\mathrm{OH}$ radicals with $\mathrm{N}$ atoms $(103 \leq T / K \leq 294)$ and with $O$ atoms (158 $\leq T / K \leq 294)$ " J. Chem. Soc. Faraday Trans., 90, 3221-3227 (1994).

[111] D. Carty, A. Goddard, S.P.K. Kohler, I.R. Sims, and I.W.M. Smith, "Kinetics of the radical-radical reaction, $O\left({ }^{3} P_{J}\right)+O H\left(X^{2} \Pi(\Omega)\right)->O_{2}+H$, at Temperatures down to $39 K^{\prime}$ " J. Phys. Chem. A, 110[9], 3101-3109 (2006).

[112] A. Bergeat, K.M. Hickson, N. Daugey, P. Caubet, and M. Costes, "A low temperature investigation of the $N\left({ }^{4} S\right)+N O$ reaction" Phys. Chem. Chem. Phys., 11[37], 8149-8155 (2009).

[113] S. Morales, C.J. Bennett, S.D. Le Picard, A. Canosa, I.R. Sims, B.J. Sun, P.H. Chen, A.H.H. Chang, V.V. Kislov, A.M. Mebel, X. Gu, F. Zhang, P. Maksyutenko, and R.I. Kaiser, "A Crossed Molecular Beams, Low Temperature Kinetics, and Theoretical Investigation of the Reaction of the Cyano Radical $(\mathrm{CN})$ with 1,3-Butadiene $\left(\mathrm{C}_{4} \mathrm{H}_{6}\right)$ - A Route to Complex Nitrogen-Bearing Molecules in Low Temperature Extraterrestrial Environments” Astrophys. J., 742[1], 26 (2011).

[114] K. McKee, M.A. Blitz, K.J. Hughes, M.J. Pilling, H.B. Qian, A. Taylor, and P.W. Seakins, " $H$ atom branching ratios from the reactions of $\mathrm{CH}$ with $\mathrm{C}_{2} \mathrm{H}_{2}, \mathrm{C}_{2} \mathrm{H}_{4}, \mathrm{C}_{2} \mathrm{H}_{6}$, and neo- $\mathrm{C}_{5} \mathrm{H}_{12}$ at room temperature and 25 torr" J. Phys. Chem. A, 107[30], 5710-5716 (2003).

[115] N. Choi, M.A. Blitz, K. McKee, M.J. Pilling, and P.W. Seakins, "H atom branching ratios from the reactions of $\mathrm{CN}$ radicals with $\mathrm{C}_{2} \mathrm{H}_{2}$ and $\mathrm{C}_{2} \mathrm{H}_{4}$ " Chem. Phys. Lett., 384[1-3], 68-72 (2004).

[116] K.L. Gannon, D.R. Glowacki, M.A. Blitz, K.J. Hughes, M.J. Pilling, and P.W. Seakins, "H atom yields from the reactions of $\mathrm{CN}$ radicals with $\mathrm{C}_{2} \mathrm{H}_{2}, \mathrm{C}_{2} \mathrm{H}_{4}, \mathrm{C}_{3} \mathrm{H}_{6}$, trans-2- $\mathrm{C}_{4} \mathrm{H}_{8}$, and iso- $\mathrm{C}_{4} \mathrm{H}_{8}$ " J. Phys. Chem. A, 111[29], 6679-6692 (2007).

[117] K.L. Gannon, M.A. Blitz, M.J. Pilling, P.W. Seakins, S.J. Klippenstein, and L.B. Harding, "Kinetics and product branching ratios of the reaction of ${ }^{1} \mathrm{C}_{2}$ with $\mathrm{H}_{2}$ and $\mathrm{D}_{2}$ " J. Phys. Chem. A, 112[39], 9575-9583 (2008).

[118] M.A. Blitz, P.W. Seakins, and I.W.M. Smith, "An experimental confirmation of the products of the reaction between $\mathrm{CN}$ radicals and $\mathrm{NH}_{3}$ " Phys. Chem. Chem. Phys., 11[46], 10824-10826 (2009). 
[119] A. Bergeat and J.C. Loison, "Reaction of carbon atoms, $C\left(2 p^{2},{ }^{3} P\right)$ with $\mathrm{C}_{2} \mathrm{H}_{2}, \mathrm{C}_{2} \mathrm{H}_{4}$ and $\mathrm{C}_{6} \mathrm{H}_{6}$ : Overall rate constant and relative atomic hydrogen production" Phys. Chem. Chem. Phys., 3[11], 2038-2042 (2001).

[120] A. Bergeat, T. Calvo, F. Caralp, J.H. Fillion, G. Dorthe, and J.C. Loison, "Determination of the $\mathrm{CH}+\mathrm{O}_{2}$ product channels" Faraday Discuss., 119, 67-77 (2001).

[121] P. Fleurat-Lessard, J.C. Rayez, A. Bergeat, and J.C. Loison, "Reaction of methylidyne CH( $\left.X^{2} \Pi\right)$ radical with $\mathrm{CH}_{4}$ and $\mathrm{H}_{2} \mathrm{~S}$ : overall rate constant and absolute atomic hydrogen production" Chem. Phys., 279[2-3], 87-99 (2002).

[122] N. Galland, F. Caralp, Y. Hannachi, A. Bergeat, and J.C. Loison, "Experimental and theoretical studies of the methylidyne $\mathrm{CH}\left(\mathrm{X}^{2} \Pi\right)$ radical reaction with ethane $\left(\mathrm{C}_{2} \mathrm{H}_{6}\right)$ : Overall rate constant and product channels" J. Phys. Chem. A, 107[28], 5419-5426 (2003).

[123] J.C. Loison and A. Bergeat, "Reaction of carbon atoms, $C\left(2 p^{2},{ }^{3} P\right)$ with $C_{3} H_{4}$ (allene and methylacetylene), $\mathrm{C}_{3} \mathrm{H}_{6}$ (propylene) and $\mathrm{C}_{4} \mathrm{H}_{8}$ (trans-butene): Overall rate constants and atomic hydrogen branching ratios" Phys. Chem. Chem. Phys., 6[23], 5396-5401 (2004).

[124] J.C. Loison, A. Bergeat, F. Caralp, and Y. Hannachi, "Rate constants and H atom branching ratios of the gas-phase reactions of methylidyne $\mathrm{CH}\left(X^{2} \Pi\right)$ radical with a series of alkanes" $\mathrm{J}$. Phys. Chem. A, 110[50], 13500-13506 (2006).

[125] J.C. Loison and A. Bergeat, "Rate constants and the H atom branching ratio of the reactions of the methylidyne $\mathrm{CH}\left(\mathrm{X}^{2} \Pi\right)$ radical with $\mathrm{C}_{2} \mathrm{H}_{2}, \mathrm{C}_{2} \mathrm{H}_{4}, \mathrm{C}_{3} \mathrm{H}_{4}$ (methylacetylene and allene), $\mathrm{C}_{3} \mathrm{H}_{6}$ (propene) and $\mathrm{C}_{4} H_{8}$ (trans-butene)" Phys. Chem. Chem. Phys., 11[4], 655-664 (2009).

[126] C.A. Taatjes and J.F. Hershberger, "Recent progress in infrared absorption techniques for elementary gas-phase reaction kinetics" Annu. Rev. Phys. Chem., 52, 41-70 (2001).

[127] F. Goulay, D.L. Osborn, C.A. Taatjes, P. Zou, G. Meloni, and S.R. Leone, "Direct detection of polyynes formation from the reaction of ethynyl radical $\left(\mathrm{C}_{2} \mathrm{H}\right)$ with propyne $\left(\mathrm{CH}_{3}-\mathrm{C} \equiv \mathrm{CH}\right)$ and allene $\left(\mathrm{CH}_{2}=\mathrm{C}=\mathrm{CH}_{2}\right)$ " Phys. Chem. Chem. Phys., 9[31], 4291-4300 (2007).

[128] T.M. Selby, G. Meloni, F. Goulay, S.R. Leone, A. Fahr, C.A. Taatjes, and D.L. Osborn, "Synchrotron photoionization mass spectrometry measurements of kinetics and product formation in the allyl radical (H2CCHCH2) self-reaction" J. Phys. Chem. A, 112[39], 93669373 (2008).

[129] F. Goulay, A.J. Trevitt, G. Meloni, T.M. Selby, D.L. Osborn, C.A. Taatjes, L. Vereecken, and S.R. Leone, "Cyclic Versus Linear Isomers Produced by Reaction of the Methylidyne Radical (CH) with Small Unsaturated Hydrocarbons" J. Amer. Chem. Soc., 131[3], 993-1005 (2009).

[130] A.J. Trevitt, F. Goulay, G. Meloni, D.L. Osborn, C.A. Taatjes, and S.R. Leone, "Isomerspecific product detection of CN radical reactions with ethene and propene by tunable VUV photoionization mass spectrometry" Int. J. Mass Spectrom., 280[1-3], 113-118 (2009).

[131] A.J. Trevitt, F. Goulay, C.A. Taatjes, D.L. Osborn, and S.R. Leone, "Reactions of the CN Radical with Benzene and Toluene: Product Detection and Low-Temperature Kinetics" J. Phys. Chem. A, 114[4], 1749-1755 (2010).

[132] S. Soorkia, A.J. Trevitt, T.M. Selby, D.L. Osborn, C.A. Taatjes, K.R. Wilson, and S.R. Leone, "Reaction of the $\mathrm{C}_{2} \mathrm{H}$ Radical with 1-Butyne $\left(\mathrm{C}_{4} \mathrm{H}_{6}\right)$ : Low-Temperature Kinetics and IsomerSpecific Product Detection" J. Phys. Chem. A, 114[9], 3340-3354 (2010). 